This is a self-archived version of an original article. This version may differ from the original in pagination and typographic details.

Author(s): Debin, Clément; Gigli, Nicola; Pasqualetto, Enrico

Title: Quasi-Continuous Vector Fields on RCD Spaces

Year: 2021

Version: Accepted version (Final draft)

Copyright: (C) Springer Nature B.V. 2020

Rights: In Copyright

Rights url: http://rightsstatements.org/page//nC/1.0/?language=en

Please cite the original version:

Debin, C., Gigli, N., \& Pasqualetto, E. (2021). Quasi-Continuous Vector Fields on RCD Spaces.

Potential Analysis, 54(1), 183-211. https://doi.org/10.1007/s11118-019-09823-6 


\title{
QUASI-CONTINUOUS VECTOR FIELDS ON RCD SPACES
}

\author{
CLÉMENT DEBIN, NICOLA GIGLI, AND ENRICO PASQUALETTO
}

\begin{abstract}
In the existing language for tensor calculus on RCD spaces, tensor fields are only defined $\mathfrak{m}$-a.e.. In this paper we introduce the concept of tensor field defined '2-capacity-a.e.' and discuss in which sense Sobolev vector fields have a 2-capacity-a.e. uniquely defined quasicontinuous representative.
\end{abstract}

\section{Contents}

Introduction 1

1. Preliminaries 3

1.1. Integration w.r.t. outer measures 3

1.2. Capacity on metric measure spaces 6

1.3. The space $L^{0}(\mathrm{Cap}) \quad 8$

1.4. Quasi-continuous functions and quasi-uniform convergence 11

2. Main result 15

2.1. $L^{0}(\mathrm{Cap})$-normed $L^{0}(\mathrm{Cap})$-modules $\quad 15$

2.2. Tangent $L^{0}$ (Cap)-module 17

2.3. Quasi-continuity of Sobolev vector fields on RCD spaces 21

References $\quad 24$

\section{INTRODUCTION}

The theory of differential calculus on RCD spaces as proposed in [9, 10] is built around the notion of $L^{0}(\mathfrak{m})$-normed $L^{0}(\mathfrak{m})$-module, which provides a convenient abstraction of the concept of 'space of measurable sections of a vector bundle'. In this sense, one thinks at such a module as the space of measurable sections of some, not really given, measurable bundle over the given metric measure space $(\mathrm{X}, \mathrm{d}, \mathfrak{m})$. More precisely, given that elements of $L^{0}(\mathfrak{m})$ are 'Borel functions identified up to equality m-a.e.', elements of such modules are, in a sense, 'measurable sections identified up to equality m-a.e.'. Notice that this interpretation is fully rigorous in the smooth case, where given a normed vector bundle, the space of its Borel sections identified up to $\mathfrak{m}$-a.e. equality is a $L^{0}(\mathfrak{m})$-normed $L^{0}(\mathfrak{m})$-module. We also remark that in $[9,10]$ the emphasis is more on the notion of $L^{\infty}(\mathfrak{m})$-module rather than on $L^{0}(\mathfrak{m})$ ones, but this is more a choice of presentation rather than an essential technical point, and given that for the purposes of this manuscript to work with $L^{0}$ is more convenient, we shall concentrate on this.

In particular, all the tensor fields on a metric measure space which are considered within the theory of $L^{0}(\mathfrak{m})$-modules are only defined $\mathfrak{m}$-a.e.. While this is an advantage in some setting, e.g. because a rigorous first order differential calculus can be built on this ground over arbitrary metric measure structures, in others is quite limiting: there certainly might be instances where, say, one 
is interested in the behaviour of a vector field on some negligible set. For instance, the question of whether the critical set of a harmonic function has capacity zero simply makes no sense if the gradient of such function only exists as element of a $L^{0}(\mathfrak{m})$-normed module.

Aim of this paper is to create a theoretical framework which allows to speak about 'Borel sections identified up to equality Cap-a.e.' and to show that Sobolev vector fields on RCD spaces, which are introduced via the theory of $L^{0}(\mathfrak{m})$-modules, in fact can be defined up to Cap-null sets and turn out to be continuous (in a sense to be made precise) outside sets of small capacities. Here the analogy is with the well-known case of Sobolev functions on the Euclidean space: these are a priori defined in a distributional-like sense, and thus up to equality $\mathcal{L}^{d}$-a.e., but once the concept of capacity is introduced one quickly realizes that a Sobolev function has a uniquelydefined representative up to Cap-null sets which is continuous outside sets of small capacities.

More in detail, in this paper we do the following:

o) We start recalling how to integrate w.r.t. an outer measure and that such integral is sublinear iff the outer measure is submodular, which is the case of capacity. This will allow to put a natural complete distance on the space $L^{0}(\mathrm{Cap})$ of real-valued Borel functions on $\mathrm{X}$ identified up to Cap-null sets. Given that Cap-null sets are $\mathfrak{m}$-null, $L^{0}(\mathfrak{m})$ can be seen as quotient of $L^{0}$ (Cap); we shall denote by $\operatorname{Pr}: L^{0}(\mathrm{Cap}) \rightarrow L^{0}(\mathfrak{m})$ the quotient map.

We then recall the concept of quasi-continuous function which, being invariant under modification on Cap-null sets, is a property of (equivalence classes of) functions in $L^{0}(\mathrm{Cap})$. The space $2 \mathcal{C}(\mathrm{X})$ of quasi-continuous functions is actually a closed subspace of $L^{0}(\mathrm{Cap})$ and coincides with the $L^{0}(\mathrm{Cap})$-closure of continuous functions (then by approximation in the uniform norm one easily sees that in proper spaces one could also take the completion of the space of locally Lipschitz functions and, in $\mathbb{R}^{d}$, of smooth ones); we believe that this characterization of quasi-continuity is well-known in the literature but have not been able to find a reference - in any case, for completeness in the preliminary section we provide full proofs of all the results we need. In connection with the concept of capacity the space $\mathcal{Q} \mathcal{C}(\mathrm{X})$ is relevant for at least two reasons:

a) The restriction of the projection operator $\operatorname{Pr}: L^{0}(\mathrm{Cap}) \rightarrow L^{0}(\mathfrak{m})$ to $\mathscr{Q} \mathcal{C}(\mathrm{X})$ is injective.

b) Any Sobolev function $f \in W^{1,2}(\mathrm{X}) \subset L^{0}(\mathfrak{m})$ has a (necessarily unique, by a) above) quasi-continuous representative $\tilde{f} \in \mathcal{Q} \mathcal{C}(\mathrm{X})$, i.e. $\operatorname{Pr}(\mathcal{Q} \mathcal{C}(\mathrm{X})) \supset W^{1,2}(\mathrm{X})$.

i) We propose the notion of $L^{0}(\mathrm{Cap})$-normed $L^{0}$ (Cap)-modules ( $L^{0}$ (Cap)-modules, in short), defined by properly imitating the one of $L^{0}(\mathfrak{m})$-module. At the technical level an important difference between the two notions is that the capacity is only an outer measure: while in some cases this is only a nuisance (see e.g. the proof of the fact that the natural distance on $L^{0}$ (Cap)-modules satisfies the triangle inequality), in others it creates problems whose solution is unclear to us (e.g. in defining the dual of a $L^{0}$ (Cap)-module - see Remark 2.3).

We then see that, much like starting from $L^{0}$ (Cap) and quotienting out up to m-null sets we find $L^{0}(\mathfrak{m})$, starting from an arbitrary $L^{0}(\mathrm{Cap})$-module $\mathscr{M}$ and quotienting via the relation

$$
v \sim w \quad \text { provided } \operatorname{Pr}(|v-w|)=0 \quad \text { m-a.e., }
$$

we produce a canonical $L^{0}(\mathfrak{m})$-module $\mathscr{M}_{\mathfrak{m}}$ and projection operator $\operatorname{Pr}_{\mathscr{M}}: \mathscr{M} \rightarrow \mathscr{M}_{\mathfrak{m}}$ (see Proposition 2.2).

ii) The main construction that we propose in this manuscript is that of tangent $L^{0}$ (Cap)module $L_{\text {Cap }}^{0}(T X)$ on an RCD space X. Specifically, in such setting we prove that there is a canonical couple $\left(L_{\text {Cap }}^{0}(T \mathrm{X}), \bar{\nabla}\right)$, where $L_{\text {Cap }}^{0}(T \mathrm{X})$ is a $L^{0}(\mathrm{Cap})$-module and $\bar{\nabla}$ : 
Test $(\mathrm{X}) \rightarrow L_{\text {Cap }}^{0}(T \mathrm{X})$ is a linear map whose image generates $L_{\text {Cap }}^{0}(T \mathrm{X})$ and such that $|\bar{\nabla} f|$ coincides with the unique quasi-continuous representative of the minimal weak upper gradient $|D f|$ of $f$, see Theorem 2.6. Here the space Test(X) of test functions is made, in some sense, of the smoothest functions available on RCD spaces; this regularity matters in the definition of $L_{\text {Cap }}^{0}(T \mathrm{X})$ to the extent that $|D f|$ belongs to $W^{1,2}(\mathrm{X})$ whenever $f \in$ Test(X) (and this fact is in turn highly depending on the lower Ricci curvature bound: it seems hard to find many functions with this property on more general metric measure spaces).

The relation between $\left(L_{\text {Cap }}^{0}(T \mathrm{X}), \bar{\nabla}\right)$ and the already known $L^{0}(\mathfrak{m})$-tangent module $L_{\mathfrak{m}}^{0}(T \mathrm{X})$ and gradient operator $\nabla$ is the fact that $L_{\mathfrak{m}}^{0}(T \mathrm{X})$ can be seen as the quotient of $L_{\text {Cap }}^{0}(T \mathrm{X})$ via the equivalence relation $(0.1)$, where the projection operator sends $\bar{\nabla} f$ to $\nabla f$ for any $f \in \operatorname{Test}(\mathrm{X})$ (see Propositions 2.9, 2.10 for the precise formulation).

iii) We define the notion of 'quasi-continuous vector field' in $L_{\text {Cap }}^{0}(T X)$. Here a relevant technical point is that there is no topology on the 'tangent bundle' or, to put it differently, it is totally unclear what it means for a tangent vector field to be continuous or continuous at a point (in fact, not even the value of a vector field at a point is defined in our setting!). In this direction we also remark that the recent result in [7] suggests that it might be pointless to look for 'many' continuous vector fields already on finite dimensional Alexandrov spaces, thus a fortiori on RCD ones.

Thus, much like in $\mathbb{R}^{d}$ quasi-continuous functions are the $L^{0}(\mathrm{Cap})$-closure of smooth ones, we define the space of quasi-continuous vector fields $\mathcal{L C}(T \mathrm{X})$ as the $L_{\text {Cap }}^{0}(T \mathrm{X})$-closure of the space of the 'smoothest' vector fields available, i.e. linear combinations of those of the form $g \tilde{\nabla} f$ for $f, g \in$ Test $(\mathrm{X})$. The choice of terminology is justified by the fact that the analogue of a), b) above hold (see Proposition 2.13 and Theorem 2.14) and, moreover:

c) for $v \in \mathcal{Q} \mathcal{C}(T \mathrm{X})$ we have $|v| \in \mathscr{Q} \mathcal{C}(\mathrm{X})$ (see Proposition 2.12).

We conclude by pointing out that, while the concept of $L^{0}(\mathrm{Cap})$-module makes sense for any $p$-capacity, for our purposes only the case $p=2$ is relevant. This is due to the fact that the natural Sobolev space to which $|D f|$ belongs for $f \in \operatorname{Test}(\mathrm{X})$ is $W^{1, p}(\mathrm{X})$ with $p=2$. Also, we remark that, albeit the definitions proposed in this paper are meant to be used in actual problems regarding the structure of RCD spaces (like the already mentioned one concerning the size of $\{\nabla f=0\}$ for $f$ harmonic - see Example 2.17 for comments in this direction), in this manuscript we concentrate on building a solid foundation of the theoretical side of the story. The added value here is in providing what we believe are the correct definitions: once these are given, proofs of relevant results will come out rather easily.

Acknowledgments This research has been supported by the MIUR SIR-grant 'Nonsmooth Differential Geometry' (RBSI147UG4).

The authors want to thank Elia Bruè and Daniele Semola for stimulating conversations on the topics of this manuscript.

\section{Preliminaries}

1.1. Integration w.r.t. outer measures. Let $\mathrm{X}$ be a given set and $\mu$ an outer measure on $\mathrm{X}$. Then for every function $f: \mathrm{X} \rightarrow[0,+\infty]$ (no measurability assumption is made here) it holds that the function $[0, \infty) \ni t \mapsto \mu(\{f>t\}) \in[0,+\infty]$ is non-increasing and thus Lebesgue measurable. 
Hence the following definition (via Cavalieri's formula)

$$
\int f \mathrm{~d} \mu:=\int_{0}^{+\infty} \mu(\{f>t\}) \mathrm{d} t .
$$

is well-posed. For an arbitrary set $E \subset \mathrm{X}$ we shall also put

$$
\int_{E} f \mathrm{~d} \mu:=\int \chi_{E} f \mathrm{~d} \mu .
$$

In the next result we collect the basic properties of the above-defined integral:

Proposition 1.1 (Basic properties of $\mu$ and integrals w.r.t. it). The following properties hold:

a) Let $f, g: \mathrm{X} \rightarrow[0,+\infty]$ be fixed. Then the following holds:

i) $\int f \mathrm{~d} \mu \leq \int g \mathrm{~d} \mu$ provided $f \leq g$.

ii) $\int \lambda f \mathrm{~d} \mu=\lambda \int f \mathrm{~d} \mu$ for every $\lambda>0$.

iii) $\int f \mathrm{~d} \mu=0$ if and only if $\mu(\{f \neq 0\})=0$.

iv) $\int f \mathrm{~d} \mu=\int g \mathrm{~d} \mu$ provided $\mu(\{f \neq g\})=0$.

v) Čebyšëv's inequality. It holds that

$$
\mu(\{f \geq \lambda\}) \leq \frac{1}{\lambda} \int_{\{f \geq \lambda\}} f \mathrm{~d} \mu \quad \text { for every } \lambda>0 .
$$

In particular, if $\int f \mathrm{~d} \mu<+\infty$ then $\mu(\{f=+\infty\})=0$.

b) Monotone convergence. Let $f, f_{n}: \mathrm{X} \rightarrow[0,+\infty], n \in \mathbb{N}$, be such that

$$
\mu\left(\left\{x \in \mathrm{X}: f_{n}(x) \ngtr f(x)\right\}\right)=0 .
$$

Then $\int f_{n} \mathrm{~d} \mu \rightarrow \int f \mathrm{~d} \mu$ as $n \rightarrow \infty$.

c) Borel-Cantelli. Let $\left(E_{n}\right)_{n \in \mathbb{N}}$ be subsets of $\mathrm{X}$ satisfying $\sum_{n \in \mathbb{N}} \mu\left(E_{n}\right)<+\infty$. Then it holds that $\mu\left(\bigcap_{n \in \mathbb{N}} \bigcup_{m \geq n} E_{m}\right)=0$.

Proof.

(a) $(i)$ is trivial and $(i i)$ follows by a change of variables. The 'if' implication in (iii) is trivial, for the 'only if' recall that $t \mapsto \mu(\{f>t\})$ is non-increasing to conclude that if $\int f \mathrm{~d} \mu=0$ it must hold $\mu(\{f>t\})=0$ for every $t>0$. Then use the countable subadditivity of $\mu$ and the identity $\{f>0\}=\bigcup_{n}\{f>1 / n\}$ to deduce that $\mu(\{f>0\})=0$. To prove $(i v)$ notice that $\{g>t\} \subset\{f>t\} \cup\{f \neq g\}$, hence taking into account the subadditivity of $\mu$ and the assumption we get $\int g \mathrm{~d} \mu \leq \int f \mathrm{~d} \mu$. Inverting the roles of $f, g$ we conclude. In order to prove $(v)$, call $E_{\lambda}:=\{f \geq \lambda\}$ and notice that $E_{\lambda} \subset\left\{\chi_{E_{\lambda}} f>t\right\}$ for all $t \in(0, \lambda)$, whence

$$
\int_{E_{\lambda}} f \mathrm{~d} \mu \geq \int_{0}^{\lambda} \mu\left(\left\{\chi_{E_{\lambda}} f>t\right\}\right) \mathrm{d} t \geq \lambda \mu\left(E_{\lambda}\right),
$$

which proves $\mu\left(E_{\lambda}\right) \leq \lambda^{-1} \int_{E_{\lambda}} f \mathrm{~d} \mu$. For the last statement, notice that if $\int f \mathrm{~d} \mu<+\infty$ then

$$
\mu(\{f=+\infty\}) \leq \underset{\lambda \rightarrow+\infty}{\lim _{\rightarrow+\infty}} \mu\left(E_{\lambda}\right) \leq \lim _{\lambda \rightarrow+\infty} \frac{1}{\lambda} \int f \mathrm{~d} \mu=0,
$$

so that $\mu(\{f=+\infty\})=0$, as required.

(b) Assume for a moment that $f_{n}(x) \uparrow f(x)$ for every $x \in \mathrm{X}$. Then the sequence of sets $\left\{f_{n}>t\right\}$ is increasing with respect to $n$ and satisfies $\bigcup_{n}\left\{f_{n}>t\right\}=\{f>t\}$. Hence the monotone convergence theorem (for the Lebesgue measure) gives

$$
\int f \mathrm{~d} \mu=\int_{0}^{+\infty} \mu(\{f>t\}) \mathrm{d} t=\lim _{n \rightarrow \infty} \int_{0}^{+\infty} \mu\left(\left\{f_{n}>t\right\}\right) \mathrm{d} t=\lim _{n \rightarrow \infty} \int f_{n} \mathrm{~d} \mu .
$$

The general case follows taking into account point $(i v)$. 
(c) The standard proof applies: let us put $E:=\bigcap_{n \in \mathbb{N}} \bigcup_{m \geq n} E_{m}$, then $\mu(E) \leq \mu\left(\bigcup_{m \geq n} E_{m}\right)$ for all $n \in \mathbb{N}$, so that

$$
\mu(E) \leq \varliminf_{n \rightarrow \infty} \mu\left(\bigcup_{m \geq n} E_{m}\right) \leq \lim _{n \rightarrow \infty} \sum_{m \geq n} \mu\left(E_{m}\right)=0
$$

as required.

Example 1.2. Consider the closed interval $S:=[0,1]$ in $\mathbb{R}$ (equipped with Euclidean distance and Lebesgue measure). Given any $n \geq 1$, denote by $P_{n} \subseteq S$ the singleton $\{1 / n\}$. One can check that $0<\operatorname{Cap}\left(P_{n}\right)<\operatorname{Cap}(S)$, but $\operatorname{Cap}\left(S \backslash P_{n}\right)=\operatorname{Cap}(S)$. In other words, $\int \chi_{S \backslash P_{n}} \mathrm{dCap}=\int \chi_{S} \mathrm{dCap}$ and $\operatorname{Cap}\left(\left\{\chi_{S \backslash P_{n}} \neq \chi_{S}\right\}\right)>0$. This shows that - even for $\mu:=$ Cap and $f \leq g$ - the converse of item iv) of Proposition 1.1 fails.

Remark 1.3. An analogue of the dominated convergence theorem cannot hold, as shown by the following counterexample. For any $n \geq 1$, consider the singleton $P_{n}$ in $\mathbb{R}$ defined in Example 1.2. Since the capacity in the space $\mathbb{R}$ is translation-invariant, one has that $\operatorname{Cap}\left(P_{n}\right)=\operatorname{Cap}\left(P_{1}\right)>0$ for all $n \geq 1$. Moreover, we have $\lim _{n} \chi_{P_{n}}(x)=0$ for all $x \in \mathbb{R}$ and $\chi_{P_{n}} \leq \chi_{[0,1]}$ for all $n \geq 1$, with $\int \chi_{[0,1]} \mathrm{dCap}=\operatorname{Cap}([0,1])<+\infty$. Nevertheless, it holds that $\int \chi_{P_{n}} \mathrm{dCap} \equiv \operatorname{Cap}\left(P_{1}\right)$ does not converge to 0 as $n \rightarrow \infty$, thus proving the failure of the dominated convergence theorem. To provide such a counterexample, we exploited the fact that the capacity is not $\sigma$-additive; indeed, we built a sequence of pairwise disjoint sets, with the same positive capacity, which are contained in a fixed set of finite capacity. The lack of a result such as the dominated convergence theorem explains the technical difficulties we will find in the proofs of Proposition 1.10 and Theorem 1.11.

Let us now introduce a crucial property of outer measures:

Definition 1.4 (Submodularity). We say that $\mu$ is submodular provided

$$
\mu(E \cup F)+\mu(E \cap F) \leq \mu(E)+\mu(F) \quad \text { for every } E, F \subset \mathrm{X} .
$$

The importance of the above notion is due to the following result (we refer to [8, Chapter 6] for a detailed bibliography):

Theorem 1.5 (Subadditivity theorem). It holds that $\mu$ is submodular if and only if the integral associated to $\mu$ is subadditive, i.e.

$$
\int(f+g) \mathrm{d} \mu \leq \int f \mathrm{~d} \mu+\int g \mathrm{~d} \mu \quad \text { for every } f, g: \mathbf{X} \rightarrow[0,+\infty) .
$$

Proof. The 'if' trivially follows by taking $f:=\chi_{E}$ and $g:=\chi_{F}$, so we turn to the 'only if'. Notice that, up to a monotone approximation argument based on point $(b)$ of Proposition 1.1, it suffices to consider the case in which $f, g$ assume only a finite number of values and $\mu(\{f>0\} \cup\{g>0\})<\infty$. Then up to replacing $\mathrm{X}$ with $\{f>0\} \cup\{g>0\}$ we can also assume that $\mu$ is finite.

Thus assume this is the case and let $\mathcal{A}$ be the (finite) algebra generated by $f, g$, i.e. the one generated by the sets $\{f=a\}$ and $\{g=b\}, a, b \in \mathbb{R}$. Let $A_{1}, \ldots, A_{n} \in \mathcal{A}$ be minimal with respect to inclusion among non-empty sets in $\mathcal{A}$ and ordered in such a way that $a_{i} \geq a_{i+1}$ for every $i=1, \ldots, n-1$, where $a_{i}:=(f+g)\left(A_{i}\right)$ (i.e. $a_{i}$ is the value of $f+g$ on $\left.A_{i}\right)$.

Define a finite measure $\nu$ on $\mathcal{A}$ by putting

$$
\nu\left(A_{1} \cup \ldots \cup A_{i}\right):=\mu\left(A_{1} \cup \ldots \cup A_{i}\right) \quad \text { for every } i=1, \ldots, n
$$

and notice that (since $\mu$ is finite) the measure $\nu$ is well-defined. We claim that for a $\mathcal{A}$-measurable function $h: \mathrm{X} \rightarrow[0,+\infty]$ it holds

$$
\int h \mathrm{~d} \mu \geq \int h \mathrm{~d} \nu \quad \text { with equality if } h\left(A_{i}\right) \geq h\left(A_{i+1}\right) \text { for every } i=1, \ldots, n-1 .
$$


Notice that once such claim is proved the conclusion easily follows from $\int(f+g) \mathrm{d} \mu=\int(f+g) \mathrm{d} \nu$ (from the equality case of the claim (1.6) and the choice of enumeration of the $A_{i}$ 's), the inequalities $\int f \mathrm{~d} \nu \leq \int f \mathrm{~d} \mu, \int g \mathrm{~d} \nu \leq \int g \mathrm{~d} \mu$ (from the claim (1.6)) and the linearity of the integral w.r.t. $\nu$.

Also, notice that the equality case in (1.6) is a direct consequence of Cavalieri's formula for both $\mu, \nu$, the defining property (1.5) and the fact that for $h$ as stated it holds $\{h>t\}=A_{1} \cup \ldots \cup A_{i}$ for some $i=i(t)$.

Let us now assume that for some $\bar{i}$ it holds $h\left(A_{\bar{i}}\right)=: b_{\bar{i}} \leq b_{\bar{i}+1}:=h\left(A_{\bar{i}+1}\right)$ and let us define another finite measure $\tilde{\nu}$ as in $(1.5)$ with the sets $\left(A_{i}\right)_{i}$ replaced by $\left(\tilde{A}_{i}\right)_{i}$, where $\tilde{A}_{i}:=A_{i}$ for $i \neq \bar{i}, \bar{i}+1, \tilde{A}_{\bar{i}}:=A_{\bar{i}+1}$ and $\tilde{A}_{\bar{i}+1}:=A_{\bar{i}}$. We shall prove that

$$
\int h \mathrm{~d} \nu \leq \int h \mathrm{~d} \tilde{\nu}
$$

and notice that this will give the proof, as with a finite number of such permutations the sets $\left(A_{i}\right)_{i}$ get ordered as in the equality case in (1.6). Using Cavalieri's formula and noticing that by construction it holds $\nu(\{h>t\})=\tilde{\nu}(\{h>t\})$ for $t \notin\left[b_{\bar{i}}, b_{\bar{i}+1}\right)$ we reduce to prove that

$$
\left(b_{\bar{i}+1}-b_{\bar{i}}\right) \nu\left(B \cup A_{\bar{i}+1}\right)=\int_{b_{\bar{i}}}^{b_{\bar{i}+1}} \nu(\{h>t\}) \mathrm{d} t \leq \int_{b_{\bar{i}}}^{b_{\bar{i}+1}} \tilde{\nu}(\{h>t\}) \mathrm{d} t=\left(b_{\bar{i}+1}-b_{\bar{i}}\right) \tilde{\nu}\left(B \cup A_{\bar{i}+1}\right)
$$

where $B:=A_{1} \cup \ldots \cup A_{\bar{i}-1}$. Given that $b_{\bar{i}+1}-b_{\bar{i}} \geq 0$, the conclusion would follow if we showed that $\nu\left(B \cup A_{\bar{i}+1}\right) \leq \tilde{\nu}\left(B \cup A_{\bar{i}+1}\right)$. Since

$\nu\left(B \cup A_{\bar{i}+1}\right)=\nu\left(B \cup A_{\bar{i}} \cup A_{\bar{i}+1}\right)+\nu(B)-\nu\left(B \cup A_{\bar{i}}\right) \stackrel{(1.5)}{=} \mu\left(B \cup A_{\bar{i}} \cup A_{\bar{i}+1}\right)+\mu(B)-\mu\left(B \cup A_{\bar{i}}\right)$,

$\tilde{\nu}\left(B \cup A_{\bar{i}+1}\right)=\tilde{\nu}\left(B \cup \tilde{A}_{\bar{i}}\right) \stackrel{(1.5)}{=} \mu\left(B \cup \tilde{A}_{\bar{i}}\right)=\mu\left(B \cup A_{\bar{i}+1}\right)$,

we know from the submodularity of $\mu$ that $\nu\left(B \cup A_{\bar{i}+1}\right) \leq \tilde{\nu}\left(B \cup A_{\bar{i}+1}\right)$, as required.

1.2. Capacity on metric measure spaces. We shall be interested in a specific outer measure: the 2-capacity (to which we shall simply refer as capacity) on a metric measure space (X, d, m).

For the purposes of the current manuscript, by metric measure space we shall always mean a triple $(X, d, \mathfrak{m})$ such that

$(\mathrm{X}, \mathrm{d})$ is a complete and separable metric space,

$\mathfrak{m} \geq 0 \quad$ is a Borel measure on $(\mathrm{X}, \mathrm{d})$, finite on balls.

In this setting, starting from [6] (see also [16, 2]) there is a well-defined notion of Sobolev space $W^{1,2}(\mathrm{X}, \mathrm{d}, \mathfrak{m})$ (or, briefly, $W^{1,2}(\mathrm{X})$ ) of real-valued functions on $\mathrm{X}$, and to any $f \in W^{1,2}(\mathrm{X}, \mathrm{d}, \mathfrak{m})$ is associated a function $|D f| \in L^{2}(\mathfrak{m})$, called minimal weak upper gradient, which plays the role of the modulus of the distributional differential of $f$. For our purposes, it will be useful to recall that $W^{1,2}(\mathrm{X}, \mathrm{d}, \mathfrak{m})$ is a lattice (i.e. $f \vee g$ and $f \wedge g$ are in $W^{1,2}(\mathrm{X})$ provided $f, g \in W^{1,2}(\mathrm{X})$ ), that the minimal weak upper gradient is local, i.e.

$$
|D f|=|D g| \quad \mathfrak{m} \text {-a.e. on }\{f=g\} \text {, }
$$

that if $f: \mathrm{X} \rightarrow \mathbb{R}$ is Lipschitz with bounded support, then

$$
|D f| \leq \operatorname{lip}(f) \quad \mathfrak{m} \text {-a.e. }
$$

where we set $\operatorname{lip}(f)(x):=\varlimsup_{y \rightarrow x}|f(y)-f(x)| / \mathrm{d}(x, y)$ if $x$ is not isolated, 0 otherwise.

Finally, the norm on $W^{1,2}(\mathrm{X})$ is defined as

$$
\|f\|_{W^{1,2}(\mathrm{X})}^{2}:=\|f\|_{L^{2}(\mathfrak{m})}^{2}+\||D f|\|_{L^{2}(\mathfrak{m})}^{2}
$$


and with this norm $W^{1,2}(\mathrm{X})$ is always a Banach space whose norm is $L^{2}$-lower semicontinuous, i.e.

$$
f_{n} \rightarrow f \text { in } L^{2}(\mathfrak{m}) \quad \Longrightarrow \quad\|f\|_{W^{1,2}(\mathrm{X})} \leq \underline{\lim }_{n \rightarrow \infty}\left\|f_{n}\right\|_{W^{1,2}(\mathrm{X})}
$$

where as customary $\|f\|_{W^{1,2}(\mathrm{X})}$ is set to be $+\infty$ if $f \notin W^{1,2}(\mathrm{X})$.

Even if in general $W^{1,2}(\mathrm{X})$ is not Hilbert (and thus the map $f \mapsto \frac{1}{2} \int|D f|^{2} \mathrm{dm}$ is not a Dirichlet form), the concept of capacity is well-defined as the definition carries over quite naturally (see e.g. [3], [12] and references therein for the metric setting, and [4] for the more classical framework of Dirichlet forms):

Definition 1.6 (Capacity). Let $E$ be a given subset of $\mathrm{X}$. Let us denote

$$
\mathcal{F}_{E}:=\left\{f \in W^{1,2}(\mathrm{X}) \mid f \geq 1 \mathfrak{m} \text {-a.e. on some open neighbourhood of } E\right\} \text {. }
$$

Then the capacity of the set $E$ is defined as the quantity $\operatorname{Cap}(E) \in[0,+\infty]$, given by

$$
\operatorname{Cap}(E):=\inf _{f \in \mathcal{F}_{E}}\|f\|_{W^{1,2}(\mathrm{X})}^{2}=\inf _{f \in \mathcal{F}_{E}} \int|f|^{2}+|D f|^{2} \mathrm{~d} \mathfrak{m},
$$

with the convention that $\operatorname{Cap}(E):=+\infty$ whenever the family $\mathcal{F}_{E}$ is empty.

In the following result we recall the main properties of the set-function Cap:

Proposition 1.7. The capacity Cap is a submodular outer measure on $\mathrm{X}$, which satisfies the following properties:

i) $\mathfrak{m}(E) \leq \operatorname{Cap}(E)$ for every Borel subset $E$ of $\mathrm{X}$,

ii) $\operatorname{Cap}(B)<\infty$ for any bounded set $B \subset \mathrm{X}$.

Proof. We start claiming that for any $f, g \in W^{1,2}(\mathrm{X})$ it holds

$$
\|f \vee g\|_{W^{1,2}(\mathrm{X})}^{2}+\|f \wedge g\|_{W^{1,2}(\mathrm{X})}^{2}=\|f\|_{W^{1,2}(\mathrm{X})}^{2}+\|g\|_{W^{1,2}(\mathrm{X})}^{2} .
$$

Indeed, the set $\{(f \vee g)(x),(f \wedge g)(x)\}$ coincides with the set $\{f(x), g(x)\}$ for m-a.e. $x$ and thus

$$
|f \vee g|^{2}(x)+|f \wedge g|^{2}(x)=|f|^{2}(x)+|g|^{2}(x)
$$

and, similarly, by the locality property (1.9) the set $\{|D(f \vee g)|(x),|D(f \wedge g)|(x)\}$ coincides with the set $\{|D f|(x),|D g|(x)\}$ for $\mathfrak{m}$-a.e. $x$ and thus

$$
|D(f \vee g)|^{2}(x)+|D(f \wedge g)|^{2}(x)=|D f|^{2}(x)+|D g|^{2}(x) .
$$

Adding up these two identities and integrating we obtain (1.14).

Now let $E_{1}, E_{2} \subset \mathrm{X}$ be given and notice that for $f_{i} \in \mathcal{F}_{E_{i}}, i=1,2$, it holds that $f_{1} \vee f_{2} \in \mathcal{F}_{E_{1} \cup E_{2}}$ and $f_{1} \wedge f_{2} \in \mathcal{F}_{E_{1} \cap E_{2}}$, thus

$$
\operatorname{Cap}\left(E_{1} \cup E_{2}\right)+\operatorname{Cap}\left(E_{1} \cap E_{2}\right) \leq\left\|f_{1} \vee f_{2}\right\|_{W^{1,2}(\mathrm{X})}^{2}+\left\|f_{1} \wedge f_{2}\right\|_{W^{1,2}(\mathrm{X})}^{2} \stackrel{(1.14)}{=}\left\|f_{1}\right\|_{W^{1,2}(\mathrm{X})}^{2}+\left\|f_{2}\right\|_{W^{1,2}(\mathrm{X})}^{2} .
$$

Hence passing to the infimum over $f_{i} \in \mathcal{F}_{E_{i}}$ we conclude that

$$
\operatorname{Cap}\left(E_{1} \cup E_{2}\right)+\operatorname{Cap}\left(E_{1} \cap E_{2}\right) \leq \operatorname{Cap}\left(E_{1}\right)+\operatorname{Cap}\left(E_{2}\right) .
$$

In particular, this shows that Cap is finitely subadditive and thus to conclude that it is a submodular outer measure it is sufficient to show that if $\left(E_{n}\right)_{n}$ is an increasing sequence of subsets of X it holds that $\operatorname{Cap}\left(\bigcup_{n} E_{n}\right)=\sup _{n} \operatorname{Cap}\left(E_{n}\right)$. Since trivially $\operatorname{Cap}(E) \leq \operatorname{Cap}(F)$ if $E \subset F$, it is sufficient to prove that $\operatorname{Cap}\left(\bigcup_{n} E_{n}\right) \leq \sup _{n} \operatorname{Cap}\left(E_{n}\right)$ and this is obvious if $\sup _{n} \operatorname{Cap}\left(E_{n}\right)=+\infty$.

Thus assume that $S:=\sup _{n} \operatorname{Cap}\left(E_{n}\right)<+\infty$ and assume for the moment also that the $E_{n}$ 's are open. Let $f_{n} \in \mathcal{F}_{E_{n}}$ be such that $\left\|f_{n}\right\|_{W^{1,2}(\mathrm{X})}^{2} \leq \operatorname{Cap}\left(E_{n}\right)+2^{-n} \leq S+1$. Thus in particular such sequence is bounded in $L^{2}(\mathfrak{m})$ and thus for some $n_{k} \uparrow+\infty$ we have that $f_{n_{k}} \rightarrow f$ in $L^{2}(\mathfrak{m})$ 
for some $f \in L^{2}(\mathfrak{m})$. Passing to the (weak) limit in $k$ in the inequality $f_{n_{k}} \geq \chi_{E_{n_{k}}} \geq \chi_{E_{n_{\ell}}}$ valid for $k \geq \ell$, we conclude that $f \geq \chi_{E_{n_{\ell}}}$ for every $\ell$, hence $f \geq \chi_{\cup_{n} E_{n}}$. Since the $E_{n}$ 's are open, this means that $f \in \mathcal{F}_{\cup_{n} E_{n}}$. Therefore taking into account the semicontinuity property (1.11) we deduce that

$$
\operatorname{Cap}\left(\bigcup_{n} E_{n}\right) \leq\|f\|_{W^{1,2}(\mathrm{X})}^{2} \leq \varliminf_{k \rightarrow \infty}\left\|f_{n_{k}}\right\|_{W^{1,2}(\mathrm{X})}^{2} \leq \varliminf_{k \rightarrow \infty} \operatorname{Cap}\left(E_{n_{k}}\right)+\frac{1}{2^{n_{k}}}=\lim _{n \rightarrow \infty} \operatorname{Cap}\left(E_{n}\right) .
$$

Now let us drop the assumption that the $E_{n}$ 's are open. Let $\varepsilon>0$. We use the submodularity property (1.15) and an induction argument to find an increasing sequence $\left(\Omega_{n}\right)_{n}$ of open sets such that $\Omega_{n} \supset E_{n}$ and $\operatorname{Cap}\left(\Omega_{n}\right) \leq \operatorname{Cap}\left(E_{n}\right)+\varepsilon \sum_{i \leq n} 2^{-i}$. Then taking into account what already proved for open sets we deduce that

$$
\operatorname{Cap}\left(\bigcup_{n} E_{n}\right) \leq \operatorname{Cap}\left(\bigcup_{n} \Omega_{n}\right)=\lim _{n \rightarrow \infty} \operatorname{Cap}\left(\Omega_{n}\right) \leq \lim _{n \rightarrow \infty} \operatorname{Cap}\left(E_{n}\right)+\varepsilon \sum_{i \leq n} \frac{1}{2^{i}}=\lim _{n \rightarrow \infty} \operatorname{Cap}\left(E_{n}\right)+\varepsilon
$$

and the conclusion follows from the arbitrariness of $\varepsilon>0$.

The inequality in $(i)$ trivially follows noticing that for $f \in \mathcal{F}_{E}$ it holds $f \geq 1 \mathfrak{m}$-a.e. on $E$, thus

$$
\int|f|^{2}+|D f|^{2} \mathrm{~d} \mathfrak{m} \geq \int_{E} 1 \mathrm{~d} \mathfrak{m}=\mathfrak{m}(E)
$$

so that the conclusion follows taking the infimum over $f \in \mathcal{F}_{E}$. For the statement $(i i)$ it is sufficient to recall that for any $B \subset \mathrm{X}$ bounded there is $f$ Lipschitz with bounded support that is $\geq 1$ on a neighbourhood of $B$ and that such $f$ belongs to $W^{1,2}(\mathrm{X})$.

1.3. The space $L^{0}$ (Cap). We have just seen that Cap is a submodular outer measure and in Subsection 1.1 we recalled how integration w.r.t. outer measures is defined. It makes therefore sense to consider the integral associated to Cap and that such integral is subadditive by Proposition 1.7 and Theorem 1.5. In light of this observation, the following definition is meaningful:

Definition 1.8 (The space $L^{0}(\mathrm{Cap})$ ). Given any two functions $f, g: \mathrm{X} \rightarrow \mathbb{R}$, we will say that $f=g$ in the Cap-a.e. sense provided $\operatorname{Cap}(\{f \neq g\})=0$. We define $L^{0}(\mathrm{Cap})$ as the space of all the equivalence classes - up to Cap-a.e. equality - of Borel functions on X.

We endow $L^{0}(\mathrm{Cap})$ with the following distance: pick an increasing sequence $\left(A_{k}\right)_{k}$ of open subsets of $\mathrm{X}$ with finite capacity such that for any $B \subset \mathrm{X}$ bounded there is $k \in \mathbb{N}$ with $B \subset A_{k}$ (for instance, one could pick $A_{k}:=B_{k}(\bar{x})$ for some $\bar{x} \in \mathrm{X}$ ), then let us define

$$
\mathrm{d}_{\text {Cap }}(f, g):=\sum_{k \in \mathbb{N}} \frac{1}{2^{k}\left(\operatorname{Cap}\left(A_{k}\right) \vee 1\right)} \int_{A_{k}}|f-g| \wedge 1 \mathrm{dCap} \quad \text { for every } f, g \in L^{0}(\mathrm{Cap}) .
$$

Notice that the integral $\int_{A_{k}}|f-g| \wedge 1 \mathrm{dCap}$ is well-defined, since its value does not depend on the particular representatives of $f$ and $g$, as granted by item $(i v)$ of Proposition 1.1. Moreover, we point out that the fact that $d_{C a p}$ satisfies the triangle inequality is a consequence of the subadditivity of the integral associated with the capacity.

Remark 1.9. We point out that if $\operatorname{Cap}(\mathrm{X})<+\infty$ then the choice $A_{k}:=\mathrm{X}$ for all $k \in \mathbb{N}$ is admissible in Definition 1.8.

The next result shows that, even if the choice of the particular sequence $\left(A_{k}\right)_{k}$ might affect the distance $\mathrm{d}_{\mathrm{Cap}}$, its induced topology remains unaltered.

Proposition 1.10 (Convergence in $\left.L^{0}(\mathrm{Cap})\right)$. The following holds:

- Characterization of Cauchy sequences. Let $\left(f_{n}\right)_{n} \subseteq L^{0}$ (Cap) be given. Then the following conditions are equivalent:

i) $\lim _{n, m} \mathrm{~d}_{\text {Cap }}\left(f_{n}, f_{m}\right)=0$, 
ii) $\lim _{n, m} \operatorname{Cap}\left(B \cap\left\{\left|f_{n}-f_{m}\right|>\varepsilon\right\}\right)=0$ for any $\varepsilon>0$ and any bounded set $B \subset \mathrm{X}$.

- Characterization of convergence. Let $f \in L^{0}(\mathrm{Cap})$ and $\left(f_{n}\right)_{n} \subseteq L^{0}(\mathrm{Cap})$. Then the following conditions are equivalent:

i) $\lim _{n} \mathrm{~d}_{\mathrm{Cap}}\left(f_{n}, f\right)=0$,

ii) $\lim _{n} \operatorname{Cap}\left(B \cap\left\{\left|f_{n}-f\right|>\varepsilon\right\}\right)=0$ for any $\varepsilon>0$ and any bounded set $B \subset \mathrm{X}$.

Proof. We shall only prove the characterization of Cauchy sequences, as the other claim follows by similar means.

i) $\Longrightarrow$ ii) Fix any $0<\varepsilon<1$ and a bounded set $B \subset \mathrm{X}$. Choose $k \in \mathbb{N}$ such that $B \subset A_{k}$. Given that $\mathrm{d}_{\text {Cap }}\left(f_{n}, f_{m}\right) \stackrel{n, m}{\longrightarrow} 0$, we have $\lim _{n, m} \int_{A_{k}}\left|f_{n}-f_{m}\right| \wedge 1 \mathrm{dCap}=0$. Therefore we conclude that

$$
\begin{aligned}
\varlimsup_{n, m \rightarrow \infty} \varepsilon \operatorname{Cap}\left(B \cap\left\{\left|f_{n}-f_{m}\right|>\varepsilon\right\}\right) & \leq \varlimsup_{n, m \rightarrow \infty} \varepsilon \operatorname{Cap}\left(A_{k} \cap\left\{\left|f_{n}-f_{m}\right|>\varepsilon\right\}\right) \\
& \leq \lim _{n, m \rightarrow \infty} \int_{A_{k}}\left|f_{n}-f_{m}\right| \wedge 1 \mathrm{dCap}=0 .
\end{aligned}
$$

ii) $\Longrightarrow$ i) Let $\varepsilon>0$ be fixed. Choose $k \in \mathbb{N}$ such that $2^{-k} \leq \varepsilon$. By our hypothesis, there is $\bar{n} \in \mathbb{N}$ such that $\operatorname{Cap}\left(A_{i} \cap\left\{\left|f_{n}-f_{m}\right|>\varepsilon\right\}\right) \leq \varepsilon \operatorname{Cap}\left(A_{i}\right)$ for every $n, m \geq \bar{n}$ and $i=1, \ldots, k$. Let us call $B_{i}^{n m}:=A_{i} \cap\left\{\left|f_{n}-f_{m}\right|>\varepsilon\right\}$ and $C_{i}^{n m}:=A_{i} \backslash B_{i}^{n m}$. Therefore for any $n, m \geq \bar{n}$ it holds that

$$
\begin{aligned}
\mathrm{d}_{\text {Cap }}\left(f_{n}, f_{m}\right) & \leq \sum_{i=1}^{k} \frac{1}{2^{i} \operatorname{Cap}\left(A_{i}\right)} \int_{A_{i}}\left|f_{n}-f_{m}\right| \wedge 1 \mathrm{dCap}+\sum_{i=k+1}^{\infty} \frac{1}{2^{i}} \\
& \leq \sum_{i=1}^{k} \frac{1}{2^{i} \operatorname{Cap}\left(A_{i}\right)}\left[\int_{B_{i}^{n m}}\left|f_{n}-f_{m}\right| \wedge 1 \mathrm{dCap}+\int_{C_{i}^{n m}}\left|f_{n}-f_{m}\right| \wedge 1 \mathrm{dCap}\right]+\varepsilon \\
& \leq \sum_{i=1}^{k} \frac{1}{2^{i} \operatorname{Cap}\left(A_{i}\right)}\left[\operatorname{Cap}\left(B_{i}^{n m}\right)+\varepsilon \operatorname{Cap}\left(A_{i}\right)\right]+\varepsilon \leq 3 \varepsilon,
\end{aligned}
$$

proving that $\lim _{n, m} \mathrm{~d}_{\text {Cap }}\left(f_{n}, f_{m}\right)=0$, as required.

Theorem 1.11. The metric space $\left(L^{0}(\mathrm{Cap}), \mathrm{d}_{\mathrm{Cap}}\right)$ is complete.

Proof. Let $\left(f_{n}\right)_{n}$ be a $\mathrm{d}_{\mathrm{Cap}}$-Cauchy sequence of Borel functions $f_{n}: \mathrm{X} \rightarrow \mathbb{R}$. Fix any $k \in \mathbb{N}$. Let $\left(f_{n_{i}}\right)_{i}$ be an arbitrary subsequence of $\left(f_{n}\right)_{n}$. Up to passing to a further (not relabeled) subsequence, it holds that

$$
\operatorname{Cap}\left(A_{k} \cap\left\{\left|f_{n_{i}}-f_{n_{i+1}}\right|>2^{-i}\right\}\right) \leq 2^{-i} \quad \text { for every } i \in \mathbb{N} .
$$

Let us call $F_{i}:=A_{k} \cap\left\{\left|f_{n_{i}}-f_{n_{i+1}}\right|>2^{-i}\right\}$ for every $i \in \mathbb{N}$ and $F:=\bigcap_{i \in \mathbb{N}} \bigcup_{j \geq i} F_{j}$. Given that $\sum_{i \in \mathbb{N}} \operatorname{Cap}\left(F_{i}\right)<+\infty$ by (1.17), we deduce from item c) of Proposition 1.1 that $\operatorname{Cap}(F)=0$. Notice that if $x \in A_{k} \backslash F=\bigcup_{i \in \mathbb{N}} \bigcap_{j \geq i} A_{k} \backslash F_{j}$, then there is $i \in \mathbb{N}$ such that $\left|f_{n_{j}}(x)-f_{n_{j+1}}(x)\right| \leq 2^{-j}$ for all $j \geq i$, which grants that $\left(f_{n_{i}}(x)\right)_{i} \subseteq \mathbb{R}$ is a Cauchy sequence for every $x \in A_{k} \backslash F$. Therefore we define the Borel function $g^{k}: A_{k} \rightarrow \mathbb{R}$ as

$$
g^{k}(x):= \begin{cases}\lim _{i} f_{n_{i}}(x) & \text { if } x \in A_{k} \backslash F, \\ 0 & \text { if } x \in F\end{cases}
$$

Now fix any $\varepsilon>0$. Choose $\bar{i} \in \mathbb{N}$ such that $\sum_{i \geq \bar{i}} 2^{-i} \leq \varepsilon$. If $i \geq \bar{i}$ and $x \in \bigcap_{j \geq i} A_{k} \backslash F_{j}$ (thus in particular $x \notin F)$, hence one has $\left|f_{n_{i}}(x)-g^{k}(x)\right| \leq \sum_{j \geq i}\left|f_{n_{j}}(x)-f_{n_{j+1}}(x)\right| \leq \sum_{j \geq i} 2^{-j} \leq \varepsilon$. This implies that

$$
A_{k} \cap\left\{\left|f_{n_{i}}-g^{k}\right|>\varepsilon\right\} \subseteq \bigcup_{j \geq i} F_{j} \quad \text { for every } i \geq \bar{i} .
$$


Then $\operatorname{Cap}\left(A_{k} \cap\left\{\left|f_{n_{i}}-g^{k}\right|>\varepsilon\right\}\right) \leq \sum_{j \geq i} \operatorname{Cap}\left(F_{j}\right) \leq \sum_{j \geq i} 2^{-j}$ holds for every $i \geq \bar{i}$, thus

$$
\lim _{i \rightarrow \infty} \operatorname{Cap}\left(A_{k} \cap\left\{\left|f_{n_{i}}-g^{k}\right|>\varepsilon\right\}\right)=0 \quad \text { for every } \varepsilon>0 .
$$

We proved this property for some subsequence of a given subsequence $\left(f_{n_{i}}\right)_{i}$ of $\left(f_{n}\right)_{n}$, hence this shows that

$$
\lim _{n \rightarrow \infty} \operatorname{Cap}\left(A_{k} \cap\left\{\left|f_{n}-g^{k}\right|>\varepsilon\right\}\right)=0 \quad \text { for every } \varepsilon>0 .
$$

Now let us define the Borel function $f: \mathrm{X} \rightarrow \mathbb{R}$ as $f:=\sum_{k \in \mathbb{N}} \chi_{A_{k}} g^{k}$. Notice that the identity $A_{k} \cap\left\{\left|f_{n}-f\right|>\varepsilon\right\}=A_{k} \cap\left\{\left|f_{n}-g^{k}\right|>\varepsilon\right\}$ and (1.20) yield

$$
\lim _{n \rightarrow \infty} \operatorname{Cap}\left(A_{k} \cap\left\{\left|f_{n}-f\right|>\varepsilon\right\}\right)=0 \quad \text { for every } k \in \mathbb{N} \text { and } \varepsilon>0 .
$$

Since any bounded subset of $\mathrm{X}$ is contained in the set $A_{k}$ for some $k \in \mathbb{N}$, we immediately deduce that $\lim _{n} \operatorname{Cap}\left(B \cap\left\{\left|f_{n}-f\right|>\varepsilon\right\}\right)=0$ whenever $\varepsilon>0$ and $B \subset \mathrm{X}$ is bounded. This grants that $\lim _{n} \mathrm{~d}_{\text {Cap }}\left(f_{n}, f\right)=0$ by Proposition 1.10 , thus proving that $\left(L^{0}(\mathrm{Cap}), \mathrm{d}_{\text {Cap }}\right)$ is a complete metric space and accordingly the statement.

We conclude this section with some other basic properties of the metric space $\left(L^{0}(\mathrm{Cap}), \mathrm{d}_{\mathrm{Cap}}\right)$ :

Proposition 1.12. Let $f_{n} \rightarrow f$ in $L^{0}$ (Cap). Then there exists a subsequence $n_{j} \uparrow+\infty$ such that for Cap-a.e. $x$ it holds that $f_{n_{j}}(x) \rightarrow f(x)$.

Moreover, the space $\mathrm{Sf}(\mathrm{X})$ of simple functions, which is defined as

$$
\mathrm{Sf}(\mathrm{X}):=\left\{\begin{array}{l|l}
\sum_{n=1}^{\infty} \alpha_{n} \chi_{E_{n}} & \begin{array}{l}
\left(\alpha_{n}\right)_{n} \subset \mathbb{R} \text { and }\left(E_{n}\right)_{n} \text { is some } \\
\text { partition of } \mathrm{X} \text { into Borel sets }
\end{array}
\end{array}\right\}
$$

is dense in $\left(L^{0}(\mathrm{Cap}), \mathrm{d}_{\mathrm{Cap}}\right)$.

Proof. As for the standard case of measures, let the subsequence satisfy $\mathrm{d}_{\mathrm{Cap}}\left(f_{n_{j}}, f_{n_{j+1}}\right) \leq 2^{-j}$ for all $j \in \mathbb{N}$. By the very definition of $\mathrm{d}_{\text {Cap }}$, we deduce that for every $j, k \in \mathbb{N}$ one has

$$
\int_{A_{k}}\left|f_{n_{j}}-f_{n_{j+1}}\right| \wedge 1 \mathrm{dCap} \leq \frac{c_{k}}{2^{j}}, \quad \text { where } c_{k}:=2^{k}\left(\operatorname{Cap}\left(A_{k}\right) \vee 1\right) .
$$

Calling $g_{j}(x):=\sum_{i=1}^{j-1}\left|f_{n_{i}}(x)-f_{n_{i+1}}(x)\right| \wedge 1$ for every $j \in \mathbb{N}$ and $x \in \mathrm{X}$, we see that $g_{j}(x) \uparrow g_{\infty}(x)$ for some $g_{\infty}: \mathrm{X} \rightarrow[0,+\infty]$. Given any $k \in \mathbb{N}$, we know from item b) of Proposition 1.1 (and the subadditivity of the integral associated to Cap) that

$$
\int_{A_{k}} g_{\infty} \mathrm{dCap}=\lim _{j \rightarrow \infty} \int_{A_{k}} g_{j} \mathrm{dCap} \leq \varliminf_{j \rightarrow \infty} \sum_{i=1}^{j-1} \int_{A_{k}}\left|f_{n_{i}}-f_{n_{i+1}}\right| \wedge 1 \mathrm{dCap} \stackrel{(1.22)}{\leq} c_{k} \varliminf_{j \rightarrow \infty} \sum_{i=1}^{j-1} \frac{1}{2^{i}}=c_{k} .
$$

Therefore item v) of Proposition 1.1 ensures that $g_{\infty}(x)<+\infty$ for Cap-a.e. $x \in A_{k}$, whence also for Cap-a.e. $x \in \mathrm{X}$ by arbitrariness of $k \in \mathbb{N}$. Now observe that for all $j^{\prime} \geq j$ and Cap-a.e. $x \in \mathrm{X}$ it holds that

$$
\left|f_{n_{j^{\prime}}}(x)-f_{n_{j}}(x)\right| \wedge 1 \leq \sum_{i=j}^{j^{\prime}-1}\left|f_{n_{i}}(x)-f_{n_{i+1}}(x)\right| \wedge 1=g_{j^{\prime}}(x)-g_{j}(x) \leq g_{\infty}(x)-g_{j}(x)<+\infty .
$$


By letting $j, j^{\prime} \rightarrow \infty$ in (1.23) we deduce that $\left(f_{n_{j}}(x)\right)_{j} \subset \mathbb{R}$ is Cauchy for Cap-a.e. $x \in \mathrm{X}$, thus it admits a limit $\tilde{f}(x) \in \mathbb{R}$. Again by item b) of Proposition 1.1 we know for every $k \in \mathbb{N}$ that

$$
\begin{aligned}
\int_{A_{k}}\left(g_{\infty}-g_{j}\right) \mathrm{dCap} & =\lim _{j^{\prime} \rightarrow \infty} \int_{A_{k}} \sum_{i=j}^{j^{\prime}}\left|f_{n_{i}}-f_{n_{i+1}}\right| \wedge 1 \mathrm{dCap} \\
& \leq \lim _{j^{\prime} \rightarrow \infty} \sum_{i=j}^{j^{\prime}} \int_{A_{k}}\left|f_{n_{i}}-f_{n_{i+1}}\right| \wedge 1 \mathrm{dCap} \\
& \stackrel{(1.22)}{\leq} c_{k} \lim _{j^{\prime} \rightarrow \infty} \sum_{i=j}^{j^{\prime}} \frac{1}{2^{i}}=\frac{c_{k}}{2^{j-1}} \stackrel{j}{\longrightarrow} 0 .
\end{aligned}
$$

By letting $j^{\prime} \rightarrow \infty$ in (1.23) we get $\left|\tilde{f}-f_{n_{j}}\right| \wedge 1 \leq g_{\infty}-g_{j}$ Cap-a.e., whence for any $k \in \mathbb{N}$ it holds

$$
\begin{aligned}
\int_{A_{k}}|\tilde{f}-f| \wedge 1 \mathrm{dCap} & \leq \underset{j \rightarrow \infty}{\lim _{j \rightarrow \infty}}\left[\int_{A_{k}}\left|\tilde{f}-f_{n_{j}}\right| \wedge 1 \mathrm{dCap}+\int_{A_{k}}\left|f_{n_{j}}-f\right| \wedge 1 \mathrm{dCap}\right] \\
& \leq \lim _{j \rightarrow \infty}\left[\int_{A_{k}}\left(g_{\infty}-g_{j}\right) \mathrm{dCap}+c_{k} \mathrm{~d}_{\text {Cap }}\left(f_{n_{j}}, f\right)\right] \stackrel{(1.24)}{=} 0 .
\end{aligned}
$$

This means that $\int_{A_{k}}|\tilde{f}-f| \wedge 1 \mathrm{dCap}=0$ for every $k \in \mathbb{N}$, thus accordingly $\tilde{f}=f$ holds Cap-a.e. by item iii) of Proposition 1.1. We then finally conclude that $f_{n_{j}}(x) \rightarrow f(x)$ for Cap-a.e. $x \in \mathrm{X}$.

For the second statement we argue as follows. Fix $f \in L^{0}(\mathrm{Cap})$ and $\varepsilon>0$. Choose a Borel representative $\bar{f}: \mathrm{X} \rightarrow \mathbb{R}$ of $f$. For any integer $i \in \mathbb{Z}$, let us define $E_{i}:=\bar{f}^{-1}([i \varepsilon,(i+1) \varepsilon))$. Then $\left(E_{i}\right)_{i \in \mathbb{Z}}$ constitutes a partition of $\mathrm{X}$ into Borel sets, so that $\bar{g}:=\sum_{i \in \mathbb{Z}} i \varepsilon \chi_{E_{i}}$ is a well-defined Borel function that belongs to $\mathrm{Sf}(\mathrm{X})$. Finally, it holds that $|\bar{f}(x)-\bar{g}(x)|<\varepsilon$ for every $x \in \mathrm{X}$, which grants that $\mathrm{d}_{\text {Cap }}(f, g) \leq \varepsilon$, where $g \in L^{0}(\mathrm{Cap})$ denotes the equivalence class of $\bar{g}$. Hence the statement follows.

Remark 1.13. In general, Cap-a.e. convergence does not imply convergence in $L^{0}$ (Cap), as shown by the following counterexample. Consider $P_{n}$ as in Example 1.2 for any $n \geq 1$. We have that the functions $f_{n}:=\chi_{P_{n}}$ pointwise converge to 0 as $n \rightarrow \infty$. However, it holds that

$$
\operatorname{Cap}\left([0,1] \cap\left\{\left|f_{n}\right|>1 / 2\right\}\right)=\operatorname{Cap}\left(P_{n}\right) \equiv \operatorname{Cap}\left(P_{1}\right)>0
$$

does not converge to 0 , thus we do not have $\lim _{n} \mathrm{~d}_{\text {Cap }}\left(f_{n}, 0\right)=0$ by Proposition 1.10.

1.4. Quasi-continuous functions and quasi-uniform convergence. Here we quickly recall the definition and main properties of quasi-continuous functions associated to Sobolev functions (see [3], [13], [4] for more on the topic and detailed bibliography).

Definition 1.14 (Quasi-continuous functions). We say that a function $f: \mathrm{X} \rightarrow \mathbb{R}$ is quasicontinuous provided for every $\varepsilon>0$ there exists a set $E \subset \mathrm{X}$ with $\operatorname{Cap}(E)<\varepsilon$ such that the function $f_{\left.\right|_{\mathrm{X} \backslash E}}: \mathrm{X} \backslash E \rightarrow \mathbb{R}$ is continuous.

It is clear that if $f, \tilde{f}$ agree Cap-a.e. and one of them is quasi-continuous, so is the other. Also, by the very definition of capacity, in defining quasi-continuity one could restrict to sets $E$ which are open. In particular, if $f$ is quasi-continuous there is an increasing sequence $\left(C_{n}\right)_{n}$ of closed subsets of $\mathrm{X}$ with $\lim _{n} \operatorname{Cap}\left(\mathrm{X} \backslash C_{n}\right)=0$ such that $f$ is continuous on each $C_{n}$. Then $N:=\bigcap_{n} \mathrm{X} \backslash C_{n}$ is a Borel set with null capacity - in particular, we have $\mathfrak{m}(N)=0$ by item i) of Proposition 1.7 - and $f$ is Borel on $\mathrm{X} \backslash N$. This proves that any quasi-continuous function is $\mathfrak{m}$-measurable and Cap-a.e. equivalent to a Borel function. 
We shall denote by $\mathcal{Q} \mathcal{C}(\mathrm{X})$ the collection of all equivalence classes - up to Cap-a.e. equality - of quasi-continuous functions on $\mathrm{X}$. What we just said ensures that $\mathcal{Q} \mathcal{C}(\mathrm{X}) \subset L^{0}(\mathrm{Cap})$. It is readily verified that $\mathcal{Q} \mathcal{C}(\mathrm{X})$ is an algebra.

Let us now discuss a notion of convergence particularly relevant in relation with $\mathcal{Q C}(\mathrm{X})$ :

Definition 1.15 (Local quasi-uniform convergence). Let $f_{n}: \mathrm{X} \rightarrow \mathbb{R}, n \in \mathbb{N} \cup\{\infty\}$ be Borel functions. Then we say that $f_{n}$ locally quasi-uniformly converges to $f_{\infty}$ as $n \rightarrow \infty$ provided for any $B \subset \mathrm{X}$ bounded and any $\varepsilon>0$ there exists a set $E \subset \mathrm{X}$ with $\operatorname{Cap}(E)<\varepsilon$ such that $f_{n} \rightarrow f_{\infty}$ uniformly on $B \backslash E$. In this case, we shall write $f_{n} \stackrel{\text { Qu }}{\rightarrow} f_{\infty}$.

As before, nothing changes if one even requires the set $E$ to be open in the above definition and the notion of local quasi-uniform convergence is invariant under modification of the functions in Cap-null sets. Local quasi-uniform convergence is (almost) the convergence induced by the following distance:

$$
\mathrm{d}_{\mathcal{Q} u}(f, g):=\inf _{E \subset \mathrm{X}} \sum_{k \in \mathbb{N}}\left(\frac{\operatorname{Cap}\left(E \cap A_{k}\right)}{2^{k}\left(\operatorname{Cap}\left(A_{k}\right) \vee 1\right)}+\frac{1}{2^{k}} \sup _{x \in A_{k} \backslash E}|f(x)-g(x)| \wedge 1\right),
$$

where $\left(A_{k}\right)_{k}$ is any sequence as in Definition 1.8. Indeed, it is trivial to verify that $\mathrm{d}_{\mathcal{Q} u}$ is actually a distance (notice that $\mathrm{d}_{\varrho} u \leq 1$, as one can see by picking $E=\mathrm{X}$ in (1.25)), moreover we have:

Proposition 1.16. Let $f_{n}: \mathrm{X} \rightarrow \mathbb{R}, n \in \mathbb{N} \cup\{\infty\}$ be Borel functions. Then

i) If $f_{n} \stackrel{\mathrm{Qu}}{\rightarrow} f_{\infty}$, then $\mathrm{d}_{\mathrm{Qu}}\left(f_{n}, f_{\infty}\right) \rightarrow 0$.

ii) If $\mathrm{d}_{\mathcal{Q} u}\left(f_{n}, f_{\infty}\right) \rightarrow 0$, then any subsequence $n_{k}$ has a further subsequence, not relabeled, such that $f_{n_{k}} \stackrel{\mathrm{Qu}}{\rightarrow} f_{\infty}$.

Proof.

(i) Let $\varepsilon>0$ and use the definition of local quasi-uniform convergence to find some subsets $\left(E_{k}\right)_{k \in \mathbb{N}}$ of X such that $\operatorname{Cap}\left(E_{k}\right)<\varepsilon / 2^{k}$ and $f_{n} \rightarrow f_{\infty}$ uniformly on $A_{k} \backslash E_{k}$ for any $k \in \mathbb{N}$. Choosing the set $E:=\bigcup_{k \in \mathbb{N}} E_{k}$ in (1.25) yields (for $\bar{k} \in \mathbb{N}$ sufficiently big)

$$
\begin{aligned}
\varlimsup_{n \rightarrow \infty} \mathrm{d}_{Q u}\left(f_{n}, f_{\infty}\right) & \leq \varepsilon+\varlimsup_{n \rightarrow \infty} \sum_{k=1}^{\bar{k}}\left(\frac{\operatorname{Cap}\left(E \cap A_{k}\right)}{2^{k}\left(\operatorname{Cap}\left(A_{k}\right) \vee 1\right)}+\frac{1}{2^{k}} \sup _{x \in A_{k} \backslash E}\left|f_{n}(x)-f_{\infty}(x)\right| \wedge 1\right) \\
& \leq \varepsilon+\varlimsup_{n \rightarrow \infty} \sum_{k=1}^{\bar{k}}\left(\frac{\varepsilon}{2^{k}}+\sup _{x \in A_{k} \backslash E_{k}}\left|f_{n}(x)-f_{\infty}(x)\right|\right) \\
& \leq 2 \varepsilon+\sum_{k=1}^{\bar{k}} \varlimsup_{n \rightarrow \infty} \sup _{x \in A_{k} \backslash E_{k}}\left|f_{n}(x)-f_{\infty}(x)\right|=2 \varepsilon,
\end{aligned}
$$

and the conclusion follows by the arbitrariness of $\varepsilon$.

(ii) We shall prove that if $\sum_{n} \mathrm{~d}_{\varrho} u\left(f_{n}, f_{\infty}\right)<+\infty$ then $f_{n} \stackrel{\varrho u}{\rightarrow} f_{\infty}$. First, choose a sequence $\left(E_{n}\right)_{n}$ of subsets of $\mathrm{X}$ such that

$$
\sum_{n \in \mathbb{N}} \sum_{k \in \mathbb{N}}\left(\frac{\operatorname{Cap}\left(E_{n} \cap A_{k}\right)}{2^{k}\left(\operatorname{Cap}\left(A_{k}\right) \vee 1\right)}+\frac{1}{2^{k}} \sup _{A_{k} \backslash E_{n}}\left|f_{n}-f_{\infty}\right| \wedge 1\right)<+\infty .
$$

Let $\varepsilon>0$ and $B \subset \mathrm{X}$ bounded be fixed. Pick $\bar{k} \in \mathbb{N}$ such that $B \subset A_{\bar{k}}$. Then (1.26) grants the existence of $\bar{n} \in \mathbb{N}$ with $\sum_{n \geq \bar{n}} \operatorname{Cap}\left(E_{n} \cap A_{\bar{k}}\right)<\varepsilon$, thus $E:=\bigcup_{n \geq \bar{n}} E_{n} \cap A_{\bar{k}}$ satisfies $\operatorname{Cap}(E)<\varepsilon$. Therefore we have that

$$
\sum_{n \geq \bar{n}} \sup _{B \backslash E}\left|f_{n}-f_{\infty}\right| \wedge 1 \leq \sum_{n \geq \bar{n}} \sup _{A_{\bar{k}} \backslash E_{n}}\left|f_{n}-f_{\infty}\right| \wedge 1=2^{\bar{k}} \sum_{n \geq \bar{n}} \frac{1}{2^{\bar{k}}} \sup _{A_{\bar{k}} \backslash E_{n}}\left|f_{n}-f_{\infty}\right| \wedge 1 \stackrel{(1.26)}{<}+\infty,
$$

whence accordingly $f_{n} \rightarrow f_{\infty}$ uniformly on $B \backslash E$. This grants that $f_{n} \stackrel{\mathrm{Qu}}{\rightarrow} f_{\infty}$, as required. 
Proposition 1.17. The following properties hold:

i) The metric space $\left(\mathcal{Q C}(\mathrm{X}), \mathrm{d}_{Q} \mathrm{u}\right)$ is complete.

ii) It holds that $\mathrm{d}_{\mathrm{Cap}}(f, g) \leq \mathrm{d}_{Q u}(f, g) \leq 2 \sqrt{\mathrm{d}_{\text {Cap }}(f, g)}$ for every $f, g \in \mathcal{Q}(\mathrm{X})$. In particular, the canonical embedding of $\mathcal{Q}(\mathrm{X})$ in $L^{0}(\mathrm{Cap})$ is continuous and has closed image.

iii) $\mathcal{Q}(\mathrm{X})$ is the closure in $L^{0}(\mathrm{Cap})$ of the space of (equivalence classes up to Cap-null sets of) continuous functions.

Proof.

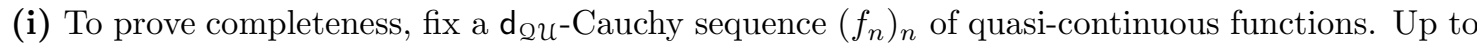
passing to a (not relabeled) subsequence, we can suppose that $\mathrm{d}_{2 u}\left(f_{n}, f_{n+1}\right)<2^{-n}$ for all $n$. For any $n \in \mathbb{N}$ we can pick a set $E_{n} \subset \mathrm{X}$ such that the function $f_{n}$ is continuous on $\mathrm{X} \backslash E_{n}$ and

$$
\sum_{k \in \mathbb{N}}\left(\frac{\operatorname{Cap}\left(E_{n} \cap A_{k}\right)}{2^{k}\left(\operatorname{Cap}\left(A_{k}\right) \vee 1\right)}+\frac{1}{2^{k}} \sup _{A_{k} \backslash E_{n}}\left|f_{n}-f_{n+1}\right| \wedge 1\right)<\frac{1}{2^{n}} .
$$

Now define $F_{k, m}:=\bigcup_{n \geq m} E_{n} \cap A_{k}$ and $F_{k}:=\bigcap_{m \in \mathbb{N}} F_{k, m}$ for every $k, m \in \mathbb{N}$. Hence one has

$$
\sum_{n \geq m} \sup _{A_{k} \backslash F_{k, m}}\left|f_{n}-f_{n+1}\right| \wedge 1 \leq \sum_{n \geq m} \sup _{A_{k} \backslash E_{n}}\left|f_{n}-f_{n+1}\right| \wedge 1 \stackrel{(1.27)}{<} 2^{k} \sum_{n \geq m} \frac{1}{2^{n}}<+\infty
$$

for any given $k, m \in \mathbb{N}$, so that $f_{n} \stackrel{n}{\rightarrow} g_{m}$ uniformly on the set $A_{k} \backslash F_{k, m}$ for some continuous function $g_{k, m}: A_{k} \backslash F_{k, m} \rightarrow \mathbb{R}$. Let us set

$$
f_{\infty}(x):= \begin{cases}g_{k, m}(x) & \text { if } x \in A_{k} \backslash F_{k, m} \text { for some } k, m \in \mathbb{N}, \\ 0 & \text { if } x \in \mathrm{X} \backslash\left(\bigcup_{k \in \mathbb{N}} A_{k} \backslash F_{k}\right) .\end{cases}
$$

Clearly $f_{\infty}$ is well-defined as $g_{k, m}=g_{k^{\prime}, m^{\prime}}$ on $\left(A_{k} \backslash F_{k, m}\right) \cap\left(A_{k^{\prime}} \backslash F_{k^{\prime}, m^{\prime}}\right)$ for all $k, k^{\prime}, m, m^{\prime} \in \mathbb{N}$. Moreover, we know from (1.27) that $\sum_{n \in \mathbb{N}} \operatorname{Cap}\left(E_{n} \cap A_{k}\right) \leq 2^{k}\left(\operatorname{Cap}\left(A_{k}\right) \vee 1\right) \sum_{n \in \mathbb{N}} 2^{-n}<+\infty$ holds for every $k \in \mathbb{N}$, whence item c) of Proposition 1.1 ensures that $\operatorname{Cap}\left(F_{k}\right)=0$ for all $k \in \mathbb{N}$. By item b) of Proposition 1.1 we see that

$$
\begin{aligned}
\operatorname{Cap}\left(\mathrm{X} \backslash\left(\bigcup_{k \in \mathbb{N}} A_{k} \backslash F_{k}\right)\right) & =\lim _{j \rightarrow \infty} \operatorname{Cap}\left(A_{j} \backslash\left(\bigcup_{k \in \mathbb{N}} A_{k} \backslash F_{k}\right)\right) \leq \varliminf_{j \rightarrow \infty} \operatorname{Cap}\left(A_{j} \backslash\left(A_{j} \backslash F_{j}\right)\right) \\
& =\lim _{j \rightarrow \infty} \operatorname{Cap}\left(F_{j}\right)=0,
\end{aligned}
$$

which shows that the function $f_{\infty}$ is quasi-continuous. We claim that $f_{n} \stackrel{2 u}{\rightarrow} f_{\infty}$, which is enough to conclude by item i) of Proposition 1.16. Given any $\varepsilon>0$ and any bounded set $B \subset \mathrm{X}$, we can pick $\bar{k}, \bar{n} \in \mathbb{N}$ such that $B \subset A_{\bar{k}}$ and $\operatorname{Cap}(E)<\varepsilon$, where we set $E:=E_{\bar{n}} \cap A_{\bar{k}}$. Therefore we have

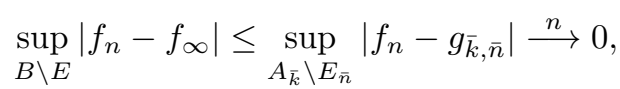

which implies that $f_{n} \stackrel{Q \mathcal{Q U}}{\rightarrow} f_{\infty}$, as desired.

(ii) Fix $f, g \in \mathcal{Q} \mathcal{C}(\mathrm{X})$ and take $\left(A_{k}\right)_{k}$ as in Definition 1.8. Given any $E \subset \mathrm{X}$, it holds that

$$
\begin{aligned}
\int_{A_{k}}|f-g| \wedge 1 \mathrm{dCap} & \leq \int_{E \cap A_{k}}|f-g| \wedge 1 \mathrm{dCap}+\int_{A_{k} \backslash E}|f-g| \wedge 1 \mathrm{dCap} \\
& \leq \operatorname{Cap}\left(E \cap A_{k}\right)+\operatorname{Cap}\left(A_{k}\right) \sup _{A_{k} \backslash E}|f-g| \wedge 1,
\end{aligned}
$$

whence accordingly

$$
\frac{1}{2^{k}\left(\operatorname{Cap}\left(A_{k}\right) \vee 1\right)} \int_{A_{k}}|f-g| \wedge 1 \mathrm{~d} \operatorname{Cap} \leq \frac{\operatorname{Cap}\left(E \cap A_{k}\right)}{2^{k}\left(\operatorname{Cap}\left(A_{k}\right) \vee 1\right)}+\frac{1}{2^{k}} \sup _{A_{k} \backslash E}|f-g| \wedge 1
$$

for every $k \in \mathbb{N}$. By summing over $k \in \mathbb{N}$ and then passing to the infimum over $E \subset \mathrm{X}$, we conclude that $\mathrm{d}_{\mathrm{Cap}}(f, g) \leq \mathrm{d}_{Q u}(f, g)$. 
On the other hand, let us consider the set $E_{\lambda}:=\{|f-g| \wedge 1>\lambda\}$ for any $\lambda>0$. Therefore for every $k \in \mathbb{N}$ one has that $\operatorname{Cap}\left(E_{\lambda} \cap A_{k}\right) \leq \lambda^{-1} \int_{A_{k}}|f-g| \wedge 1$ dCap by item v) of Proposition 1.1 and that $\sup _{A_{k} \backslash E_{\lambda}}|f-g| \wedge 1 \leq \lambda$, thus accordingly

$$
\begin{aligned}
\mathrm{d}_{\Omega u}(f, g) & \leq \sum_{k \in \mathbb{N}}\left(\frac{\operatorname{Cap}\left(E_{\lambda} \cap A_{k}\right)}{2^{k}\left(\operatorname{Cap}\left(A_{k}\right) \vee 1\right)}+\frac{1}{2^{k}} \sup _{x \in A_{k} \backslash E_{\lambda}}|f(x)-g(x)| \wedge 1\right) \\
& \leq \lambda+\frac{1}{\lambda} \sum_{k \in \mathbb{N}} \frac{1}{2^{k}\left(\operatorname{Cap}\left(A_{k}\right) \vee 1\right)} \int_{A_{k}}|f-g| \wedge 1 \mathrm{dCap}=\lambda+\frac{\mathrm{d}_{\text {Cap }}(f, g)}{\lambda} .
\end{aligned}
$$

By letting $\lambda \downarrow \sqrt{\mathrm{d}_{\text {Cap }}(f, g)}$ we conclude that $\mathrm{d}_{\mathcal{Q}}(f, g) \leq 2 \sqrt{\mathrm{d}_{\text {Cap }}(f, g)}$, as required.

(iii) Let $f: \mathrm{X} \rightarrow \mathbb{R}$ be a Borel function whose equivalence class up to Cap-null sets belongs to $\mathrm{QC}(\mathrm{X})$ and $\varepsilon>0$. Then by definition there is an open set $\Omega$ with $\operatorname{Cap}(\Omega)<\varepsilon$ and $f_{\left.\right|_{\mathrm{X} \backslash \Omega}}$ is continuous. By the Tietze extension theorem there is $g \in C(\mathrm{X})$ which agrees with $f$ on $\mathrm{X} \backslash \Omega$, and - since this latter condition ensures that $\mathrm{d}_{Q}(f, g)<\varepsilon$ - the proof is achieved.

We now turn to the relation between quasi-continuity and Sobolev functions, and to do so it is useful to emphasise whether we speak about functions up to Cap-null sets or up to m-null sets. We shall therefore write $[f]_{\text {Cap }}$ (resp. $[f]_{\mathfrak{m}}$ ) for the equivalence class of the Borel function $f: \mathrm{X} \rightarrow \mathbb{R}$ up to Cap-null (resp. m-null) sets.

We start noticing that - since $\mathfrak{m}$ is absolutely continuous with respect to Cap, i.e. Cap-null sets are also $\mathfrak{m}$-null (recall $(i)$ of Proposition 1.7) - there is a natural projection map

$$
\begin{aligned}
\operatorname{Pr}: L^{0}(\mathrm{Cap}) & \longrightarrow L^{0}(\mathfrak{m}), \\
{[f]_{\text {Cap }} } & \longmapsto[f]_{\mathfrak{m}} .
\end{aligned}
$$

Since in general there are $\mathfrak{m}$-null sets which are not Cap-null, such projection operator is typically non-injective. This is why the following result is interesting:

Proposition 1.18 (Uniqueness of quasi-continuous representative). Let $f, g: \mathrm{X} \rightarrow \mathbb{R}$ be quasicontinuous functions. Then $f=g$ m-a.e. implies $f=g$ Cap-a.e.. In other words,

$$
\operatorname{Pr}_{\left.\right|_{\mathcal{L}(\mathrm{X})}}: \operatorname{Qe}(\mathrm{X}) \longrightarrow L^{0}(\mathfrak{m})
$$

is an injective map.

Proof. Let $N:=\{f \neq g\}$. Let $\Omega \subset \mathrm{X}$ open be such that $f, g$ are continuous on $\mathrm{X} \backslash \Omega$. Thus $N$ is open in $\mathrm{X} \backslash \Omega$ and therefore $\tilde{\Omega}:=N \cup \Omega$ is open in X. By assumption we know that $\mathfrak{m}(N)=0$ and thus the very definition of capacity yields $\operatorname{Cap}(\Omega)=\operatorname{Cap}(\tilde{\Omega})$. Hence

$$
\operatorname{Cap}(N) \leq \operatorname{Cap}(\tilde{\Omega})=\operatorname{Cap}(\Omega)
$$

and the quasi-continuity assumption gives the conclusion.

Proposition 1.19. Let $f, g \in C(\mathrm{X})$ be such that $[f]_{\mathfrak{m}},[g]_{\mathfrak{m}} \in W^{1,2}(\mathrm{X})$. Then

$$
\mathrm{d}_{\mathcal{Q U}}\left([f]_{\text {Cap }},[g]_{\text {Cap }}\right) \leq 3\left\|[f]_{\mathfrak{m}}-[g]_{\mathfrak{m}}\right\|_{W^{1,2}(\mathrm{X})}^{\frac{2}{3}} .
$$

Proof. For any $\lambda>0$ let $\Omega_{\lambda}:=\{|f-g|>\lambda\}$, so that by definition of $\mathrm{d}_{\varrho u}$ we have

$$
\mathrm{d}_{\mathcal{Q}}\left([f]_{\text {Cap }},[g]_{\text {Cap }}\right) \leq \lambda+\sum_{k \in \mathbb{N}} \frac{\operatorname{Cap}\left(\Omega_{\lambda} \cap A_{k}\right)}{2^{k}\left(\operatorname{Cap}\left(A_{k}\right) \vee 1\right)} .
$$

Notice that $\Omega_{\lambda}$ is an open set by continuity of $|f-g|$. Moreover, $\lambda^{-1}[|f-g|]_{\mathfrak{m}}$ is a Sobolev function satisfying $\lambda^{-1}[|f-g|]_{\mathfrak{m}} \geq 1 \mathfrak{m}$-a.e. on $\Omega_{\lambda}$. Hence $\operatorname{Cap}\left(\Omega_{\lambda} \cap A_{k}\right) \leq \lambda^{-2}\left\|[f-g]_{\mathfrak{m}}\right\|_{W^{1,2}(\mathrm{X})}^{2}$ 
holds for all $k \in \mathbb{N}$. Plugging this estimate in (1.29) we obtain that

$$
\mathrm{d}_{\mathcal{Q U}}\left([f]_{\mathrm{Cap}},[g]_{\mathrm{Cap}}\right) \leq \lambda+2 \frac{\left\|[f]_{\mathfrak{m}}-[g]_{\mathfrak{m}}\right\|_{W^{1,2}(\mathrm{X})}^{2}}{\lambda^{2}},
$$

then by choosing $\lambda:=\left\|[f]_{\mathfrak{m}}-[g]_{\mathfrak{m}}\right\|_{W^{1,2}(\mathrm{X})}^{\frac{2}{3}}$ we get the conclusion.

Collecting these last two propositions we obtain the following result:

Theorem 1.20 (Quasi-continuous representative of Sobolev function). Suppose that (equivalence classes up to $\mathfrak{m}$-a.e. equality of) continuous functions in $W^{1,2}(\mathrm{X})$ are dense in $W^{1,2}(\mathrm{X})$. Then there exists a unique continuous map

$$
\mathrm{QCR}: W^{1,2}(\mathrm{X}) \longrightarrow \mathrm{Q} \mathrm{C}(\mathrm{X})
$$

such that the composition $\operatorname{Pr} \circ \mathrm{QCR}$ is the inclusion map $W^{1,2}(\mathrm{X}) \subset L^{0}(\mathfrak{m})$.

Moreover, QCR is linear and satisfies

$$
|\mathrm{QCR}(f)|=\mathrm{QCR}(|f|) \quad \text { Cap-a.e. for every } f \in W^{1,2}(\mathrm{X}) \text {. }
$$

Finally, if $\left[f_{n}\right]_{\mathfrak{m}} \rightarrow[f]_{\mathfrak{m}}$ in $W^{1,2}(\mathrm{X})$, then any subsequence has a further subsequence converging locally quasi-uniformly.

Proof. For $f \in C(\mathrm{X})$ with $[f]_{\mathfrak{m}} \in W^{1,2}(\mathrm{X})$ the requirements for $\mathrm{QCR}(f)$ are that it must belong to $\mathcal{Q C}(\mathrm{X})$ and satisfy $\operatorname{Pr}(\mathrm{QCR}(f))=[f]_{\mathfrak{m}}$. Thus Proposition 1.18 forces it to be equal to $[f]_{\mathrm{Cap}}$. Proposition 1.19 ensures that such assignment is Lipschitz as map from $W^{1,2}(\mathrm{X}) \cap C(\mathrm{X})$ to $\mathcal{Q} \mathcal{C}(\mathrm{X})$, and thus can be uniquely extended to a continuous map on the whole $W^{1,2}(\mathrm{X})$.

Since QCR is linear on continuous functions, by continuity it is linear on the whole $W^{1,2}(\mathrm{X})$. (1.31) is trivial for continuous functions, thus its validity for general ones follows by continuity.

The last statement is a direct consequence of what already proved and Proposition 1.16.

\section{MAIN RESUlt}

2.1. $L^{0}(\mathrm{Cap})$-normed $L^{0}(\mathrm{Cap})$-modules. The language of $L^{0}(\mathfrak{m})$-normed $L^{0}(\mathfrak{m})$-modules over a metric measure space $(\mathrm{X}, \mathrm{d}, \mathfrak{m})$ has been proposed and investigated by the second author in [9], with the final aim of developing a differential calculus on RCD spaces. In the present paper, we assume the reader to be familiar with such language. We shall use the term $L^{0}(\mathfrak{m})$-module in place of $L^{0}(\mathfrak{m})$-normed $L^{0}(\mathfrak{m})$-module and we will typically denote by $\mathscr{M}_{\mathfrak{m}}$ any such object. We refer to $[9,10]$ for a detailed account about this topic. Here we introduce a new notion of normed module, called $L^{0}(\mathrm{Cap})$-normed $L^{0}(\mathrm{Cap})$-module or, more simply, $L^{0}(\mathrm{Cap})$-module, in which the measure under consideration is the capacity Cap instead of the reference measure $\mathfrak{m}$.

Let $(\mathrm{X}, \mathrm{d}, \mathfrak{m})$ be a metric measure space as in $(1.8)$ and $\left(A_{k}\right)_{k}$ a sequence as in Definition 1.8.

Definition 2.1 ( $L^{0}(\mathrm{Cap})$-normed $L^{0}$ (Cap)-module). We say that a quadruple $(\mathscr{M}, \tau, \cdot,|\cdot|)$ is a $L^{0}$ (Cap)-normed $L^{0}(\mathrm{Cap})$-module over $(\mathrm{X}, \mathrm{d}, \mathfrak{m})$ provided:

i) $(\mathscr{M}, \tau)$ is a topological vector space.

ii) The bilinear map $\cdot: L^{0}(\mathrm{Cap}) \times \mathscr{M} \rightarrow \mathscr{M}$ satisfies $f \cdot(g \cdot v)=(f g) \cdot v$ and $1 \cdot v=v$ for every $f, g \in L^{0}(\mathrm{Cap})$ and $v \in \mathscr{M}$.

iii) The $\operatorname{map}|\cdot|: \mathscr{M} \rightarrow L^{0}$ (Cap), called pointwise norm, satisfies

$$
\begin{aligned}
|v| \geq 0 & \text { for every } v \in \mathscr{M}, \text { with equality if and only if } v=0, \\
|v+w| \leq|v|+|w| & \text { for every } v, w \in \mathscr{M}, \\
|f \cdot v|=|f||v| & \text { for every } v \in \mathscr{M} \text { and } f \in L^{0}(\mathrm{Cap}),
\end{aligned}
$$


where all equalities and inequalities are intended in the Cap-a.e. sense.

iv) The distance $\mathrm{d}_{\mathscr{M}}$ on $\mathscr{M}$, given by

$$
\mathrm{d}_{\mathscr{M}}(v, w):=\sum_{k \in \mathbb{N}} \frac{1}{2^{k}\left(\operatorname{Cap}\left(A_{k}\right) \vee 1\right)} \int_{A_{k}}|v-w| \wedge 1 \mathrm{dCap} \quad \text { for all } v, w \in \mathscr{M},
$$

is complete and induces the topology $\tau$.

Much like starting from $L^{0}(\mathrm{Cap})$ and passing to the quotient up to $\mathfrak{m}$-a.e. equality we obtain $L^{0}(\mathfrak{m})$, in the same way by passing to an appropriate quotient starting from an arbitrary $L^{0}(\mathrm{Cap})$ module we obtain a $L^{0}(\mathfrak{m})$-module. Let us describe this procedure.

Let $\mathscr{M}$ be a $L^{0}$ (Cap)-module and define an equivalence relation on it by declaring

$$
v \sim_{\mathfrak{m}} w \Longleftrightarrow|v-w|=0 \text { m-a.e. in } \mathrm{X} .
$$

Then we consider the quotient $\mathscr{M}_{\mathfrak{m}}:=\mathscr{M} / \sim_{\mathfrak{m}}$, the projection map $\operatorname{Pr}_{\mathscr{M}}: \mathscr{M} \rightarrow \mathscr{M}_{\mathfrak{m}}$ sending $v$ to its equivalence class $[v]_{\mathfrak{m}}$ and define the following operations on $\mathscr{M}_{\mathfrak{m}}$ :

$$
\begin{aligned}
{[v]_{\mathfrak{m}}+[w]_{\mathfrak{m}} } & :=[v+w]_{\mathfrak{m}} \in \mathscr{M}_{\mathfrak{m}}, \\
{[f]_{\mathfrak{m}} \cdot[v]_{\mathfrak{m}} } & :=\left[[f]_{\mathrm{Cap}} \cdot v\right]_{\mathfrak{m}} \in \mathscr{M}_{\mathfrak{m}}, \\
\left|[v]_{\mathfrak{m}}\right| & :=\operatorname{Pr}(|v|) \in L^{0}(\mathfrak{m}),
\end{aligned}
$$

for every $v, w \in \mathscr{M}$ and $f: \mathrm{X} \rightarrow \mathbb{R}$ Borel, where $\operatorname{Pr}: L^{0}(\mathrm{Cap}) \rightarrow L^{0}(\mathfrak{m})$ is the projection operator defined in (1.28). Routine verifications show that with these operations $\mathscr{M}_{\mathfrak{m}}$ is a $L^{0}(\mathfrak{m})$-module.

For a given $L^{0}(\mathrm{Cap})$-module $\mathscr{M}$, the couple $\left(\mathscr{M}_{\mathfrak{m}}, \operatorname{Pr}_{\mathscr{M}}\right)$ is characterized by the following universal property:

Proposition 2.2 (Universal property of $\left(\mathscr{M}_{\mathfrak{m}}, \operatorname{Pr}_{\mathscr{M}}\right)$ ). Let $\mathscr{M}$ be a $L^{0}(\mathrm{Cap})$-module and let $\left(\mathscr{M}_{\mathfrak{m}}, \operatorname{Pr}_{\mathscr{M}}\right)$ be defined as above. Also, let $\mathscr{N}_{\mathfrak{m}}$ be a $L^{0}(\mathfrak{m})$-module and $T: \mathscr{M} \rightarrow \mathscr{N}_{\mathfrak{m}}$ be a linear map satisfying

$$
|T(v)| \leq \operatorname{Pr}(|v|) \quad \mathfrak{m} \text {-a.e. for every } v \in \mathscr{M} .
$$

Then there is a unique $L^{0}(\mathfrak{m})$-linear and continuous map $T_{\mathrm{Pr}}: \mathscr{M}_{\mathfrak{m}} \rightarrow \mathscr{N}_{\mathfrak{m}}$ such that the diagram

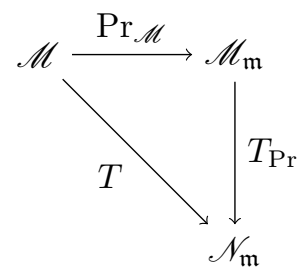

commutes.

In particular, for any other couple $\left(\mathscr{M}_{\mathfrak{m}}^{\prime}, \operatorname{Pr}_{\mathscr{M}}^{\prime}\right)$ with the same property there is a unique isomorphism $\Phi: \mathscr{M}_{\mathfrak{m}} \rightarrow \mathscr{M}_{\mathfrak{m}}^{\prime}$ (i.e. bijection which preserves the whole structure of $L^{0}$ (Cap)-module) such that $\Phi \circ \operatorname{Pr}_{\mathscr{M}}=\operatorname{Pr}_{\mathscr{M}}^{\prime}$.

Proof. The latter statement is an obvious consequence of the former, so we concentrate on this one. Let $v, w \in \mathscr{M}$ be such that $v \sim_{\mathfrak{m}} w$ and notice that

$$
|T(v)-T(w)|=|T(v-w)| \stackrel{(2.1)}{\leq} \operatorname{Pr}(|v-w|)=0 \quad \text { holds } \mathfrak{m} \text {-a.e.. }
$$

Thus $T$ passes to the quotient and defines a map $T_{\operatorname{Pr}}: \mathscr{M}_{\mathfrak{m}} \rightarrow \mathscr{N}_{\mathfrak{m}}$ making the diagram (2.2) commute. It is clear that $T_{\mathrm{Pr}}$ is linear and continuous (the latter being a consequence of (2.1) and the definition), thus to conclude it is sufficient to prove $L^{0}(\mathfrak{m})$-linearity. By linearity and 
continuity this will follow if we show that $T_{\operatorname{Pr}}\left(\left[\chi_{E}\right]_{\mathfrak{m}}[v]_{\mathfrak{m}}\right)=\left[\chi_{E}\right]_{\mathfrak{m}} T_{\operatorname{Pr}}\left([v]_{\mathfrak{m}}\right)$ for any Borel set $E \subset \mathrm{X}$; in turn, this will follow if we prove that

$$
T\left(\left[\chi_{E}\right]_{\text {Cap }} v\right)=\left[\chi_{E}\right]_{\mathfrak{m}} T(v) \quad \text { for every } v \in \mathscr{M} \text { and } E \subset \mathrm{X} \text { Borel. }
$$

To show this, notice that from (2.1) it follows $\left|T\left(\left[\chi_{E^{c}}\right]_{\text {Cap }} v\right)\right| \leq\left[\chi_{E^{c}}\right]_{\mathfrak{m}} \operatorname{Pr}(|v|)$, thus multiplying both sides by $\left[\chi_{E}\right]_{\mathfrak{m}}$ we obtain

$$
\left[\chi_{E}\right]_{\mathfrak{m}} T\left(\left[\chi_{E^{c}}\right]_{\text {Cap }} v\right)=0 \text { and symmetrically } \quad\left[\chi_{E^{c}}\right]_{\mathfrak{m}} T\left(\left[\chi_{E}\right]_{\text {Cap }} v\right)=0 .
$$

Therefore

$$
\begin{aligned}
T\left(\left[\chi_{E}\right]_{\text {Cap }} v\right) & =\left(\left[\chi_{E}\right]_{\mathfrak{m}}+\left[\chi_{E^{c}}\right]_{\mathfrak{m}}\right) T\left(\left[\chi_{E}\right]_{\text {Cap }} v\right) \\
& \stackrel{(2.3)}{=}\left[\chi_{E}\right]_{\mathfrak{m}} T\left(\left[\chi_{E}\right]_{\text {Cap }} v\right) \\
& \stackrel{(2.3)}{=}\left[\chi_{E}\right]_{\mathfrak{m}}\left(T\left(\left[\chi_{E}\right]_{\text {Cap }} v\right)+T\left(\left[\chi_{E^{c}}\right]_{\text {Cap }} v\right)\right)=\left[\chi_{E}\right]_{\mathfrak{m}} T(v)
\end{aligned}
$$

and the conclusion follows.

Remark 2.3. In analogy with the case of $L^{0}(\mathfrak{m})$-modules, one could be tempted to define the dual of a $L^{0}$ (Cap)-module $\mathscr{M}_{\text {Cap }}$ as the space of $L^{0}$ (Cap)-linear continuous maps $L: \mathscr{M}_{\text {Cap }} \rightarrow L^{0}$ (Cap) and to declare that the pointwise norm $|L|$ of any such $L$ is the minimal element of $L^{0}$ (Cap) (where minimality is intended in the Cap-a.e. sense) such that the inequality $|L| \geq L(v)$ holds Cap-a.e. for any $v \in \mathscr{M}_{\text {Cap }}$ that Cap-a.e. satisfies $|v| \leq 1$.

Technically speaking, for $L^{0}(\mathfrak{m})$-modules this can be achieved by using the notion of essential supremum of a family of Borel functions. Nevertheless, it seems that this tool cannot be adapted to the situation in which we want to consider the capacity instead of the reference measure, as suggested by Example 1.2.

Definition 2.4. Let $\mathscr{H}$ be a $L^{0}(\mathrm{Cap})$-module over $(\mathrm{X}, \mathrm{d}, \mathfrak{m})$. Then we say that $\mathscr{H}$ is a Hilbert module provided

$$
|v+w|^{2}+|v-w|^{2}=2|v|^{2}+2|w|^{2} \quad \text { holds Cap-a.e. in } \mathrm{X}
$$

for every $v, w \in \mathscr{H}$.

By polarisation, we define a pointwise scalar product $\langle\cdot, \cdot\rangle: \mathscr{H} \times \mathscr{H} \rightarrow L^{0}(\mathrm{Cap})$ as

$$
\langle v, w\rangle:=\frac{|v+w|^{2}-|v|^{2}-|w|^{2}}{2} \quad \text { Cap-a.e. in X. }
$$

Then the operator $\langle\cdot, \cdot\rangle$ is $L^{0}(\mathrm{Cap})$-bilinear and satisfies

$$
\begin{aligned}
|\langle v, w\rangle| & \leq|v||w| \quad \text { Cap-a.e. for every } v, w \in \mathscr{H} . \\
\langle v, v\rangle & =|v|^{2} \quad \text {. }
\end{aligned}
$$

2.2. Tangent $L^{0}(\mathrm{Cap})$-module. Let $(\mathrm{X}, \mathrm{d}, \mathfrak{m})$ be an $\operatorname{RCD}(K, \infty)$ space, for some $K \in \mathbb{R}$. A fundamental class of Sobolev functions on $\mathrm{X}$ is that of test functions, denoted by Test(X) (cf. [9]). We point out that we are in a position to apply Theorem 1.20 above, since Lipschitz functions with bounded support are dense in $W^{1,2}(\mathrm{X})$, as proven in [1]. Moreover, a fact that is fundamental for our discussion (see [15]) is the following:

$$
\langle\nabla f, \nabla g\rangle \in W^{1,2}(\mathrm{X}) \quad \text { for every } f, g \in \operatorname{Test}(\mathrm{X}) .
$$

In particular, by taking $g=f$ in (2.7) we get $|D f|^{2} \in W^{1,2}(\mathrm{X})$ for every $f \in \operatorname{Test}(\mathrm{X})$.

Let us use the notation $L_{\mathfrak{m}}^{0}(T \mathrm{X})$ to indicate the tangent $L^{0}(\mathfrak{m})$-module over $(\mathrm{X}, \mathrm{d}, \mathfrak{m})$. Recall that $\operatorname{TestV}(\mathrm{X}) \subseteq L_{\mathfrak{m}}^{0}(T \mathrm{X})$ denotes the class of test vector fields on $\mathrm{X}$, while $H_{C}^{1,2}(T \mathrm{X})$ is the closure 
of $\operatorname{TestV}(\mathrm{X})$ in the Sobolev space $W_{C}^{1,2}(T \mathrm{X})$. We know from [10, Proposition 2.19] that for any $v \in H_{C}^{1,2}(T \mathrm{X}) \cap L_{\mathfrak{m}}^{\infty}(T \mathrm{X})$ one has that $|v|^{2} \in W^{1,2}(\mathrm{X})$ and

$$
\mathrm{d}|v|^{2}(w)=2\left\langle\nabla_{w} v, v\right\rangle \quad \mathfrak{m} \text {-a.e. } \quad \text { for every } w \in L_{\mathfrak{m}}^{0}(T X),
$$

whence in particular $\left.\left.|D| v\right|^{2}|\leq 2| \nabla v\right|_{\text {HS }}|v|$ holds $\mathfrak{m}$-a.e.. This in turn implies the following:

Lemma 2.5. Let $(\mathrm{X}, \mathrm{d}, \mathfrak{m})$ be an $\operatorname{RCD}(K, \infty)$ space, for some $K \in \mathbb{R}$. Let $v \in H_{C}^{1,2}(T \mathrm{X})$ be fixed. Then $|v| \in W^{1,2}(\mathrm{X})$ and

$$
|D| v|| \leq|\nabla v|_{\mathrm{HS}} \quad \text { holds } \mathfrak{m} \text {-a.e. in } \mathrm{X} .
$$

Proof. First of all, we prove the statement for $v \in \operatorname{TestV}(\mathrm{X})$. Given any $\varepsilon>0$, let us define the Lipschitz function $\varphi_{\varepsilon}:[0,+\infty) \rightarrow \mathbb{R}$ as $\varphi_{\varepsilon}(t):=\sqrt{t+\varepsilon}$ for any $t \geq 0$. Hence by applying the chain rule for minimal weak upper gradients we see that $\varphi_{\varepsilon} \circ|v|^{2} \in \mathrm{S}^{2}$ (X) (cf. [2] for the notion of Sobolev class $\left.\mathrm{S}^{2}(\mathrm{X})\right)$ and

$$
\left|D\left(\varphi_{\varepsilon} \circ|v|^{2}\right)\right|=\left.\left.\varphi_{\varepsilon}^{\prime} \circ|v|^{2}|D| v\right|^{2}\left|=\frac{\left.|D| v\right|^{2} \mid}{2 \sqrt{|v|^{2}+\varepsilon}} \leq \frac{|v|}{\sqrt{|v|^{2}+\varepsilon}}\right| \nabla v\right|_{\mathrm{HS}} \leq|\nabla v|_{\mathrm{HS}} .
$$

This grants the existence of $G \in L^{2}(\mathfrak{m})$ and a sequence $\varepsilon_{j} \searrow 0$ such that $\left|D\left(\varphi_{\varepsilon_{j}} \circ|v|^{2}\right)\right| \rightarrow G$ weakly in $L^{2}(\mathfrak{m})$ as $j \rightarrow \infty$ and $G \leq|\nabla v|_{\text {HS }}$ in the $\mathfrak{m}$-a.e. sense. Since $\varphi_{\varepsilon_{j}} \circ|v|^{2} \rightarrow|v|$ pointwise $\mathfrak{m}$-a.e. as $j \rightarrow \infty$, we deduce from the lower semicontinuity of minimal weak upper gradients that $|v| \in W^{1,2}(\mathrm{X})$ and that $|D| v|| \leq|\nabla v|_{\text {HS }}$ holds $\mathfrak{m}$-a.e. in $\mathrm{X}$.

Now fix $v \in H_{C}^{1,2}(T \mathrm{X})$. Pick a sequence $\left(v_{n}\right)_{n} \subseteq \operatorname{TestV}(\mathrm{X})$ that $W_{C}^{1,2}(T \mathrm{X})$-converges to $v$. In particular, $\left|v_{n}\right| \rightarrow|v|$ and $\left|\nabla v_{n}\right|_{\text {HS }} \rightarrow|\nabla v|_{\text {HS }}$ in $L^{2}(\mathfrak{m})$. By the first part of the proof we know that $\left|v_{n}\right| \in W^{1,2}(\mathrm{X})$ and $|D| v_{n}|| \leq\left|\nabla v_{n}\right|_{\text {HS }}$ for all $n \in \mathbb{N}$, thus accordingly (up to a not relabeled subsequence) we have that $|D| v_{n}|| \rightarrow H$ weakly in $L^{2}(\mathfrak{m})$, for some $H \in L^{2}(\mathfrak{m})$ such that $H \leq|\nabla v|_{\text {HS }}$ holds $\mathfrak{m}$-a.e. in $\mathrm{X}$. Again by lower semicontinuity of minimal weak upper gradients we conclude that $|v| \in W^{1,2}(\mathrm{X})$ with $|D| v|| \leq|\nabla v|_{\text {HS }}$ in the $\mathfrak{m}$-a.e. sense, proving the statement.

We now introduce the so-called tangent $L^{0}(\mathrm{Cap})$-module $L_{\mathrm{Cap}}^{0}(T \mathrm{X})$ over $\mathrm{X}$, which is a $L^{0}(\mathrm{Cap})$ module in the sense of Definition 2.1.

Theorem 2.6 (Tangent $L^{0}(\mathrm{Cap})$-module). Let $(\mathrm{X}, \mathrm{d}, \mathfrak{m})$ be an $\operatorname{RCD}(K, \infty)$ space. Then there exists a unique couple $\left(L_{\mathrm{Cap}}^{0}(T \mathrm{X}), \bar{\nabla}\right)$, where $L_{\mathrm{Cap}}^{0}(T \mathrm{X})$ is a $L^{0}(\mathrm{Cap})$-module over $\mathrm{X}$ and the operator $\bar{\nabla}: \operatorname{Test}(\mathrm{X}) \rightarrow L_{\mathrm{Cap}}^{0}(T \mathrm{X})$ is linear, such that the following properties hold:

i) For any $f \in \operatorname{Test}(\mathrm{X})$ we have that the equality $|\bar{\nabla} f|=\mathrm{QCR}(|D f|)$ holds Cap-a.e. on $\mathrm{X}$ (note that $|D f| \in W^{1,2}(\mathrm{X})$ as a consequence of Lemma 2.5).

ii) The space of $\sum_{n \in \mathbb{N}} \chi_{E_{n}} \bar{\nabla} f_{n}$, with $\left(f_{n}\right)_{n} \subseteq \operatorname{Test}(\mathrm{X})$ and $\left(E_{n}\right)_{n}$ Borel partition of $\mathrm{X}$, is dense in $L_{\text {Cap }}^{0}(T \mathrm{X})$.

Uniqueness is intended up to unique isomorphism: given another couple $\left(\mathscr{M}_{\mathrm{Cap}}, \bar{\nabla}^{\prime}\right)$ with the same properties, there exists a unique isomorphism $\Phi: L_{\text {Cap }}^{0}(T \mathrm{X}) \rightarrow \mathscr{M}_{\text {Cap }}$ with $\Phi \circ \bar{\nabla}=\bar{\nabla}^{\prime}$.

The space $L_{\mathrm{Cap}}^{0}(T \mathrm{X})$ is called tangent $L^{0}(\mathrm{Cap})$-module associated to $(\mathrm{X}, \mathrm{d}, \mathfrak{m})$, while its elements are said to be Cap-vector fields on X. Moreover, the operator $\bar{\nabla}$ is called gradient.

Proof.

Uniqueness. Consider any simple vector field $v \in L_{\text {Cap }}^{0}(T X)$, i.e. $v=\sum_{n \in \mathbb{N}} \chi_{E_{n}} \bar{\nabla} f_{n}$ for some $\left(f_{n}\right)_{n} \subseteq \operatorname{Test}(\mathrm{X})$ and $\left(E_{n}\right)_{n}$ Borel partition of $\mathrm{X}$. We are thus forced to set

$$
\Phi(v):=\sum_{n \in \mathbb{N}} \chi_{E_{n}} \bar{\nabla}^{\prime} f_{n} \in \mathscr{M}_{\text {Cap }}
$$


Such definition is well-posed, as granted by the Cap-a.e. equalities

$$
\left|\sum_{n \in \mathbb{N}} \chi_{E_{n}} \bar{\nabla}^{\prime} f_{n}\right|=\sum_{n \in \mathbb{N}} \chi_{E_{n}}\left|\bar{\nabla}^{\prime} f_{n}\right|=\sum_{n \in \mathbb{N}} \chi_{E_{n}}\left|D f_{n}\right|=\sum_{n \in \mathbb{N}} \chi_{E_{n}}\left|\bar{\nabla} f_{n}\right|=|v|,
$$

which also show that $\Phi$ preserves the pointwise norm of simple vector fields. In particular, the map $\Phi$ is linear and continuous, whence it can be uniquely extended to a linear and continuous operator $\Phi: L_{\text {Cap }}^{0}(T X) \rightarrow \mathscr{M}_{\text {Cap }}$ by density of simple vector fields in $L_{\text {Cap }}^{0}(T X)$. It follows from Proposition 1.12 that $\Phi$ preserves the pointwise norm. Moreover, we know from the definition (2.10) that $\Phi(f v)=f \Phi(v)$ is satisfied for any simple $f$ and $v$, whence also for all $f \in L^{0}(\mathrm{Cap})$ and $v \in L_{\mathrm{Cap}}^{0}(T \mathrm{X})$ by Proposition 1.12. To conclude, just notice that the image of $\Phi$ is dense in $\mathscr{M}_{\text {Cap }}$ by density of simple vector fields in $\mathscr{M}_{\text {Cap }}$, thus accordingly $\Phi$ is surjective (as its image is closed, being $\Phi$ an isometry). Therefore we proved that there exists a unique module isomorphism $\Phi: L_{\text {Cap }}^{0}(T X) \rightarrow \mathscr{M}_{\text {Cap }}$ such that $\Phi \circ \bar{\nabla}=\bar{\nabla}^{\prime}$, as required.

Existence. We define the 'pre-tangent module' Ptm as the set of all sequences $\left(E_{n}, f_{n}\right)_{n}$, where $\left(f_{n}\right)_{n} \subseteq \operatorname{Test}(\mathrm{X})$ and $\left(E_{n}\right)_{n}$ is a Borel partition of $\mathrm{X}$. We now define an equivalence relation $\sim$ on Ptm: we declare that $\left(E_{n}, f_{n}\right)_{n} \sim\left(F_{m}, g_{m}\right)_{m}$ provided

$$
\mathrm{QCR}\left(\left|D\left(f_{n}-g_{m}\right)\right|\right)=0 \quad \text { Cap-a.e. on } E_{n} \cap F_{m} \quad \text { for every } n, m \in \mathbb{N} \text {. }
$$

The equivalence class of $\left(E_{n}, f_{n}\right)_{n}$ will be denoted by $\left[E_{n}, f_{n}\right]_{n}$. Moreover, let us define

$$
\alpha\left[E_{n}, f_{n}\right]_{n}+\beta\left[F_{m}, g_{m}\right]_{m}:=\left[E_{n} \cap F_{m}, \alpha f_{n}+\beta g_{m}\right]_{n, m}
$$

for every $\alpha, \beta \in \mathbb{R}$ and $\left[E_{n}, f_{n}\right]_{n},\left[F_{m}, g_{m}\right]_{m} \in \mathrm{Ptm} / \sim$, so that $\mathrm{Ptm} / \sim$ inherits a vector space structure; well-posedness of these operations is granted by the locality property of minimal weak upper gradients and by Theorem 1.20. We define the pointwise norm of any given element $\left[E_{n}, f_{n}\right]_{n} \in \mathrm{Ptm} / \sim$ as

$$
\left|\left[E_{n}, f_{n}\right]_{n}\right|:=\sum_{n \in \mathbb{N}} \chi_{E_{n}} \mathrm{QCR}\left(\left|D f_{n}\right|\right) \in L^{0}(\mathrm{Cap})
$$

Then we define $L_{\text {Cap }}^{0}(T \mathrm{X})$ as the completion of the metric space $\left(\operatorname{Ptm} / \sim, \mathrm{d}_{L_{\text {Cap }}^{0}}(T \mathrm{X})\right)$, where

$$
\mathrm{d}_{L_{\text {Cap }}^{0}(T \mathrm{X})}(v, w):=\sum_{k \in \mathbb{N}} \frac{1}{2^{k}\left(\operatorname{Cap}\left(A_{k}\right) \vee 1\right)} \int_{A_{k}}|v-w| \wedge 1 \mathrm{dCap} \quad \text { for all } v, w \in \operatorname{Ptm} / \sim,
$$

while we set $\bar{\nabla} f:=[\mathrm{X}, f] \in L_{\mathrm{Cap}}^{0}(T \mathrm{X})$ for every test function $f \in \operatorname{Test}(\mathrm{X})$, thus obtaining a linear operator $\bar{\nabla}: \operatorname{Test}(\mathrm{X}) \rightarrow L_{\mathrm{Cap}}^{0}(T \mathrm{X})$. Item i) of the statement is thus clearly satisfied. Observe that $\left[E_{n}, f_{n}\right]_{n}=\sum_{n \in \mathbb{N}} \chi_{E_{n}} \bar{\nabla} f_{n}$ for every $\left[E_{n}, f_{n}\right]_{n} \in \mathrm{Ptm} / \sim$, so that also item ii) is verified, as a consequence of the density of $\mathrm{Ptm} / \sim$ in $L_{\text {Cap }}^{0}(T X)$. Now let us define the multiplication operator $\cdot: \operatorname{Sf}(\mathrm{X}) \times(\mathrm{Ptm} / \sim) \rightarrow \mathrm{Ptm} / \sim$ as follows:

$$
\left(\sum_{m \in \mathbb{N}} \alpha_{m} \chi_{F_{m}}\right) \cdot\left[E_{n}, f_{n}\right]_{n}:=\left[E_{n} \cap F_{m}, \alpha_{m} f_{n}\right]_{n, m} \in \mathrm{Ptm} / \sim .
$$

Therefore the maps defined in (2.11) and (2.13) can be uniquely extended by continuity to a pointwise norm operator $|\cdot|: L_{\text {Cap }}^{0}(T X) \rightarrow L^{0}(\mathrm{Cap})$ and a multiplication by $L^{0}(\mathrm{Cap})$-functions • : $L^{0}(\mathrm{Cap}) \times L_{\text {Cap }}^{0}(T \mathrm{X}) \rightarrow L_{\text {Cap }}^{0}(T \mathrm{X})$, respectively. It also turns out that the distance $\mathrm{d}_{L_{\text {Cap }}^{0}}(T \mathrm{X})$ is expressed by the formula in (2.12) for any $v, w \in L_{\mathrm{Cap}}^{0}(T \mathrm{X})$, as one can readily deduce from Proposition 1.12. Finally, standard verifications show that $L_{\mathrm{Cap}}^{0}(T \mathrm{X})$ is a $L^{0}(\mathrm{Cap})$-module over $(\mathrm{X}, \mathrm{d}, \mathfrak{m})$, thus concluding the proof. 
Remark 2.7. An analogous construction has been carried out in [9] to define the cotangent $L^{0}(\mathfrak{m})$ module $L_{\mathfrak{m}}^{0}\left(T^{*} \mathrm{X}\right)$, while the tangent $L^{0}(\mathfrak{m})$-module $L_{\mathfrak{m}}^{0}(T \mathrm{X})$ was obtained from the cotangent one by duality. However, since we cannot consider duals of $L^{0}$ (Cap)-modules (as pointed out in Remark 2.3), we opted for a different axiomatisation. We just underline the fact that, since RCD spaces are infinitesimally Hilbertian, the modules $L_{\mathfrak{m}}^{0}\left(T^{*} \mathrm{X}\right)$ and $L_{\mathfrak{m}}^{0}(T \mathrm{X})$ can be canonically identified via the Riesz isomorphism.

Proposition 2.8. The tangent $L^{0}(\mathrm{Cap})$-module $L_{\mathrm{Cap}}^{0}(T \mathrm{X})$ is a Hilbert module.

Proof. Given any $f, g \in \operatorname{Test}(\mathrm{X})$, we deduce from item i) of Theorem 2.6 and the last statement of Theorem 1.20 that

$$
\begin{aligned}
|\bar{\nabla} f+\bar{\nabla} g|^{2}+|\bar{\nabla} f-\bar{\nabla} g|^{2} & =\mathrm{QCR}\left(|D(f+g)|^{2}+|D(f-g)|^{2}\right)=\mathrm{QCR}\left(2|D f|^{2}+2|D g|^{2}\right) \\
& =2|\bar{\nabla} f|^{2}+2|\bar{\nabla} g|^{2} .
\end{aligned}
$$

This grants that the pointwise parallelogram identity (2.4) is satisfied whenever $v, w$ are $L^{0}$ (Cap)linear combinations of elements of $\{\bar{\nabla} f: f \in \operatorname{Test}(\mathrm{X})\}$, whence also for any $v, w \in L_{\text {Cap }}^{0}(T \mathrm{X})$ by approximation. This proves that $L_{\text {Cap }}^{0}(T \mathrm{X})$ is a Hilbert module, as required.

We now investigate the relation that subsists between tangent $L^{0}$ (Cap)-module and tangent $L^{0}(\mathfrak{m})$-module. We start with the following result, which shows the existence of a natural projection operator $\overline{P r}$ sending $\bar{\nabla} f$ to $\nabla f$ :

Proposition 2.9. There exists a unique linear continuous operator $\overline{\operatorname{Pr}}: L_{\text {Cap }}^{0}(T \mathrm{X}) \rightarrow L_{\mathfrak{m}}^{0}(T \mathrm{X})$ that satisfies the following properties:

i) $\operatorname{Pr}(\bar{\nabla} f)=\nabla f$ for every $f \in \operatorname{Test}(\mathrm{X})$.

ii) $\overline{\operatorname{Pr}}(g v)=\operatorname{Pr}(g) \overline{\operatorname{Pr}}(v)$ for every $g \in L^{0}($ Cap $)$ and $v \in L_{\text {Cap }}^{0}(T \mathrm{X})$.

Moreover, the operator $\overline{\mathrm{Pr}}$ satisfies

$$
|\overline{\operatorname{Pr}}(v)|=\operatorname{Pr}(|v|) \quad \mathfrak{m} \text {-a.e. } \quad \text { for every } v \in L_{\text {Cap }}^{0}(T \mathrm{X}) .
$$

Proof. Given a Borel partition $\left(E_{n}\right)_{n \in \mathbb{N}}$ of $\mathrm{X}$ and $\left(v_{n}\right)_{n \in \mathbb{N}} \subseteq L_{\text {Cap }}^{0}(T \mathrm{X})$, we are forced to set

$$
\overline{\operatorname{Pr}}\left(\sum_{n \in \mathbb{N}}\left[\chi_{E_{n}}\right]_{\operatorname{Cap}} \bar{\nabla} f_{n}\right):=\sum_{n \in \mathbb{N}}\left[\chi_{E_{n}}\right]_{\mathfrak{m}} \nabla f_{n}
$$

The well-posedness of such definition stems from the following $\mathfrak{m}$-a.e. equalities:

$$
\begin{aligned}
\left|\sum_{n \in \mathbb{N}}\left[\chi_{E_{n}}\right]_{\mathfrak{m}} \nabla f_{n}\right| & =\sum_{n \in \mathbb{N}}\left[\chi_{E_{n}}\right]_{\mathfrak{m}}\left|D f_{n}\right|=\sum_{n \in \mathbb{N}} \operatorname{Pr}\left(\left[\chi_{E_{n}}\right]_{\text {Cap }}\right) \operatorname{Pr}\left(\operatorname{QCR}\left(\left|D f_{n}\right|\right)\right) \\
& =\operatorname{Pr}\left(\sum_{n \in \mathbb{N}}\left[\chi_{E_{n}}\right]_{\text {Cap }} \operatorname{QCR}\left(\left|D f_{n}\right|\right)\right)=\operatorname{Pr}\left(\sum_{n \in \mathbb{N}}\left[\chi_{E_{n}}\right]_{\text {Cap }}\left|\bar{\nabla} f_{n}\right|\right) \\
& =\operatorname{Pr}\left(\left|\sum_{n \in \mathbb{N}}\left[\chi_{E_{n}}\right]_{\text {Cap }} \bar{\nabla} f_{n}\right|\right) .
\end{aligned}
$$

Moreover, we also infer that such map $\overline{\mathrm{Pr}}$ - which is linear by construction - is also continuous, whence it admits a unique linear and continuous extension $\overline{\operatorname{Pr}}: L_{\text {Cap }}^{0}(T \mathrm{X}) \rightarrow L_{\mathfrak{m}}^{0}(T \mathrm{X})$. Property i) is clearly satisfied by (2.15). From the linearity of $\nabla$ and $\bar{\nabla}$, we deduce that property ii) holds for any simple function $g \in L^{0}(\mathrm{Cap})$, thus also for any $g \in L^{0}$ (Cap) by approximation. Finally, again by approximation we see that (2.14) follows from (2.16).

The fact that $L_{\mathrm{Cap}}^{0}(T \mathrm{X})$ can be thought of as a natural 'refinement' of the already known $L_{\mathfrak{m}}^{0}(T \mathrm{X})$ is now encoded in the following proposition, which shows that $\left(L_{\mathfrak{m}}^{0}(T \mathrm{X}), \overline{\mathrm{Pr}}\right)$ is the canonical quotient of $L_{\text {Cap }}^{0}(T X)$ up to m-a.e. equality (recall Proposition 2.2): 
Proposition 2.10. Let $\mathscr{N}_{\mathfrak{m}}$ be a $L^{0}(\mathfrak{m})$-module and $T: L_{\text {Cap }}^{0}(T \mathrm{X}) \rightarrow \mathscr{N}_{\mathfrak{m}}$ linear and such that

$$
|T(v)| \leq \operatorname{Pr}(|v|) \mathfrak{m} \text {-a.e. } \quad \text { for every } v \in L_{\text {Cap }}^{0}(T \mathrm{X}) .
$$

Then there is a unique $L^{0}(\mathfrak{m})$-linear and continuous map $S: L_{\mathfrak{m}}^{0}(T \mathrm{X}) \rightarrow \mathscr{N}_{\mathfrak{m}}$ such that the diagram

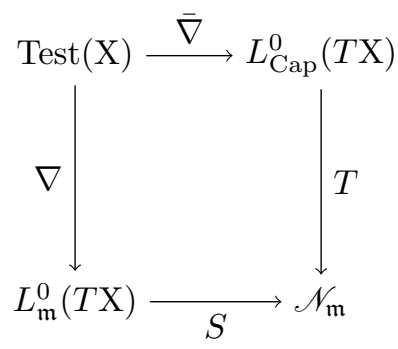

commutes.

Proof. By (2.17) it follows that $|T(\bar{\nabla} f)-T(\bar{\nabla} g)| \leq|D(f-g)|$ holds $\mathfrak{m}$-a.e. and thus

$$
f, g \in \operatorname{Test}(\mathrm{X}),|D(f-g)|=0 \quad \mathfrak{m} \text {-a.e. on } E \subset \mathrm{X} \quad \Longrightarrow \quad T(\bar{\nabla} f)=T(\bar{\nabla} g) \quad \mathfrak{m} \text {-a.e. on } E \text {. }
$$

Now let $V \subset L_{\mathfrak{m}}^{0}(T \mathrm{X})$ be the space of finite sums of the form $\sum_{i}\left[\chi_{E_{i}}\right]_{\mathfrak{m}} \nabla f_{i}$ for $\left(E_{i}\right)_{i}$ Borel subsets of $\mathrm{X}$ and $\left(f_{i}\right)_{i} \subset \operatorname{Test}(\mathrm{X})$, and define $S: V \rightarrow \mathscr{N}_{\mathfrak{m}}$ as:

$$
S(v):=\sum_{i}\left[\chi_{E_{i}}\right]_{\mathfrak{m}} T\left(\bar{\nabla} f_{i}\right) \in \mathscr{N}_{\mathfrak{m}} \quad \text { for every } v=\sum_{i}\left[\chi_{E_{i}}\right]_{\mathfrak{m}} \nabla f_{i} \in V .
$$

The implication in (2.19) grants that this is a good definition, i.e. the value of $S(v)$ depends only on $v$ and not on how it is written as finite sum of the form $\sum_{i}\left[\chi_{E_{i}}\right]_{\mathfrak{m}} \nabla f_{i}$. It is clear that $S$ is linear and that, by (2.17) and item i) of Theorem 2.6, it holds

$$
|S(v)| \leq|v| \quad \mathfrak{m} \text {-a.e. } \quad \text { for every } v \in V .
$$

In particular, $S$ is 1-Lipschitz from $V$ (with the $L_{\mathfrak{m}}^{0}(T \mathrm{X})$-distance) to $\mathscr{N}_{\mathfrak{m}}$. Since $V$ is dense in $L_{\mathfrak{m}}^{0}(T \mathrm{X}), S$ can be uniquely extended to a continuous map - still denoted by $S$ - from $L_{\mathfrak{m}}^{0}(T \mathrm{X})$ to $\mathscr{N}_{\mathfrak{m}}$. Clearly such extension is linear and, by (2.20), it also satisfies $S\left(\left[\chi_{E}\right]_{\mathfrak{m}} v\right)=\left[\chi_{E}\right]_{\mathfrak{m}} S(v)$ (e.g. by mimicking the arguments used in the proof of Proposition 2.2). These two facts easily imply $L^{0}(\mathfrak{m})$-linearity, thus showing existence of the desired map $S$. For uniqueness simply notice that the value of $S$ on the dense subspace $V$ of $L_{\mathfrak{m}}^{0}(T \mathrm{X})$ is forced by the commutativity of the diagram in (2.18).

2.3. Quasi-continuity of Sobolev vector fields on RCD spaces. Let (X,d, m) be an $\operatorname{RCD}(K, \infty)$ space, for some $K \in \mathbb{R}$. The aim of this conclusive subsection is to prove that any element of the space $H_{C}^{1,2}(T \mathrm{X})$ admits a quasi-continuous representative, in a suitable sense. We begin with the definition of quasi-continuous vector field on $\mathrm{X}$ :

Definition 2.11 (Quasi-continuity for vector fields). We define the set $\operatorname{Test} \overline{\mathrm{V}}(\mathrm{X}) \subseteq L_{\text {Cap }}^{0}(T \mathrm{X})$ as

$$
\operatorname{Test} \overline{\mathrm{V}}(\mathrm{X}):=\left\{\sum_{i=0}^{n} \mathrm{QCR}\left(g_{i}\right) \bar{\nabla} f_{i} \mid n \in \mathbb{N},\left(f_{i}\right)_{i=1}^{n},\left(g_{i}\right)_{i=1}^{n} \subseteq \operatorname{Test}(\mathrm{X})\right\} .
$$

Then the space $\mathcal{Q}(T \mathrm{X})$ of quasi-continuous vector fields on $\mathrm{X}$ is defined as the $\mathrm{d}_{L_{\mathrm{Cap}}^{0}(T \mathrm{X}) \text {-closure }}$ of $\operatorname{Test} \overline{\mathrm{V}}(\mathrm{X})$ in $L_{\mathrm{Cap}}^{0}(T \mathrm{X})$. It clearly holds that $\mathscr{Q} \mathcal{C}(T \mathrm{X})$ is a vector subspace of $L_{\text {Cap }}^{0}(T \mathrm{X})$.

Proposition 2.12. Let $v \in \mathcal{Q} \mathcal{C}(T \mathrm{X})$ be given. Then it holds that $|v| \in \mathcal{Q C}(\mathrm{X})$. 
Proof. First of all, if $v=\sum_{i=0}^{n} \mathrm{QCR}\left(g_{i}\right) \bar{\nabla} f_{i} \in \operatorname{Test} \overline{\mathrm{V}}(\mathrm{X})$ then

$$
\begin{aligned}
|v|^{2} & =\sum_{i, j=0}^{n} \mathrm{QCR}\left(g_{i}\right) \mathrm{QCR}\left(g_{j}\right)\left\langle\bar{\nabla} f_{i}, \bar{\nabla} f_{j}\right\rangle \\
& =\sum_{i, j=0}^{n} \operatorname{QCR}\left(g_{i}\right) \operatorname{QCR}\left(g_{j}\right) \frac{\left|\bar{\nabla}\left(f_{i}+f_{j}\right)\right|^{2}-\left|\bar{\nabla} f_{i}\right|^{2}-\left|\bar{\nabla} f_{j}\right|^{2}}{2} \\
& =\sum_{i, j=0}^{n} \operatorname{QCR}\left(g_{i}\right) \operatorname{QCR}\left(g_{j}\right) \frac{\operatorname{QCR}\left(\left|D\left(f_{i}+f_{j}\right)\right|^{2}\right)-\operatorname{QCR}\left(\left|D f_{i}\right|^{2}\right)-\operatorname{QCR}\left(\left|D f_{j}\right|^{2}\right)}{2} \in \mathcal{Q}(\mathrm{X}) .
\end{aligned}
$$

For general $v \in \mathcal{Q} \mathcal{C}(T \mathrm{X})$ we proceed by approximation: chosen any sequence $\left(v_{n}\right)_{n} \subset \operatorname{Test} \overline{\mathrm{V}}(\mathrm{X})$ such that $\mathrm{d}_{L_{\text {Cap }}^{0}(T \mathrm{X})}\left(v_{n}, v\right) \rightarrow 0$, or equivalently $\mathrm{d}_{\text {Cap }}\left(\left|v_{n}-v\right|, 0\right) \rightarrow 0$, we have that $\left|v_{n}\right| \rightarrow|v|$ with respect to $\mathrm{d}_{C a p}$, whence accordingly $|v| \in \mathcal{L} \mathcal{C}(\mathrm{X})$ by Proposition 1.17.

Proposition 2.13. It holds that the map $\overline{\operatorname{Pr}}_{\left.\right|_{\mathcal{Q C}(T \mathrm{X})}}: \operatorname{LC}(T \mathrm{X}) \rightarrow L_{\mathfrak{m}}^{0}(T \mathrm{X})$ is injective.

Proof. Let $v, w \in \mathcal{Q} \mathcal{C}(T \mathrm{X})$ be such that $\overline{\operatorname{Pr}}(v)=\overline{\operatorname{Pr}}(w)$. In other words, we have that

$$
\operatorname{Pr}(|v-w|) \stackrel{(2.14)}{=}|\overline{\operatorname{Pr}}(v-w)|=0 \quad \text { holds } \mathfrak{m} \text {-a.e. in } \mathrm{X},
$$

whence Proposition 1.18 grants that $|v-w|=0$ holds Cap-a.e. in X. This shows that $v=w$, thus proving the claim.

We are ready to state and prove the main result of the paper: any element of $H_{C}^{1,2}(T \mathrm{X})$ admits a quasi-continuous representative in $\mathcal{Q} \mathcal{C}(T \mathrm{X})$. This is a generalisation of Theorem 1.20 to vector fields over an RCD space.

Theorem 2.14 (Quasi-continuous representative of a Sobolev vector field). Let us fix any $\operatorname{RCD}(K, \infty)$ space $(\mathrm{X}, \mathrm{d}, \mathfrak{m})$, for some $K \in \mathbb{R}$. Then there exists a unique map

$$
\mathrm{Q} \overline{\mathrm{CR}}: H_{C}^{1,2}(T \mathrm{X}) \longrightarrow \mathrm{Q} \mathcal{C}(T \mathrm{X})
$$

such that $\overline{\mathrm{Pr}} \circ \mathrm{Q} \overline{\mathrm{CR}}: H_{C}^{1,2}(T \mathrm{X}) \rightarrow L_{\mathfrak{m}}^{0}(T \mathrm{X})$ coincides with the inclusion $H_{C}^{1,2}(T \mathrm{X}) \subset L_{\mathfrak{m}}^{0}(T \mathrm{X})$. Moreover, $\mathrm{Q} \bar{C} \mathrm{R}$ is linear and $|\mathrm{Q} \overline{\mathrm{CR}}(v)|=\mathrm{QCR}(|v|)$ holds for every $v \in H_{C}^{1,2}(T \mathrm{X})$.

Proof. Fix $v \in H_{C}^{1,2}(T \mathrm{X})$. Pick $\left(\bar{v}_{n}\right)_{n} \subseteq \operatorname{Test} \overline{\mathrm{V}}(\mathrm{X})$ such that $v_{n}:=\overline{\operatorname{Pr}}\left(\bar{v}_{n}\right) \rightarrow v$ in $W_{C}^{1,2}(T \mathrm{X})$. We know from Lemma 2.5 that $\left|v_{n}-v\right| \in W^{1,2}(\mathrm{X})$ and $|D| v_{n}-v|| \leq\left|\nabla\left(v_{n}-v\right)\right|_{\text {HS }}$ m-a.e. for all $n \in \mathbb{N}$, thus accordingly $\left|v_{n}-v\right| \rightarrow 0$ in $W^{1,2}(\mathrm{X})$ as $n \rightarrow \infty$. Proposition 1.19 grants that - up to a (not relabeled) subsequence - we have that QCR $\left(\left|v_{n}-v\right|\right) \rightarrow 0$ locally quasi-uniformly as $n \rightarrow \infty$, whence QCR $\left(\left|v_{n}-v_{m}\right|\right) \rightarrow 0$ locally quasi-uniformly as $n, m \rightarrow \infty$. Thus Proposition 1.17 yields

$$
\mathrm{d}_{L_{\text {Cap }}^{0}(T \mathrm{X})}\left(\bar{v}_{n}, \bar{v}_{m}\right)=\mathrm{d}_{\mathrm{Cap}}\left(\mathrm{QCR}\left(\left|v_{n}-v_{m}\right|\right), 0\right) \longrightarrow 0 \quad \text { as } n, m \rightarrow \infty .
$$

This shows that $\left(\bar{v}_{n}\right)_{n} \subseteq L_{\text {Cap }}^{0}(T \mathrm{X})$ is Cauchy, thus it converges to some $\bar{v} \in L_{\text {Cap }}^{0}(T \mathrm{X})$. Hence one has $\overline{\operatorname{Pr}}(\bar{v})=\overline{\operatorname{Pr}}\left(\lim _{n} \bar{v}_{n}\right)=\lim _{n} \overline{\operatorname{Pr}}\left(\bar{v}_{n}\right)=\lim _{n} v_{n}=v$, so that we define $\mathrm{Q} \overline{C R}(v):=\bar{v}$. Proposition 2.13 grants that the map QC $\mathrm{C}: H_{C}^{1,2}(T \mathrm{X}) \rightarrow \mathcal{Q} \mathcal{C}(T \mathrm{X})$ is well-defined and is the unique map such that $\overline{\operatorname{Pr}} \circ \mathrm{Q} \bar{C}$ coincides with the inclusion $H_{C}^{1,2}(T \mathrm{X}) \subset L_{\mathfrak{m}}^{0}(T \mathrm{X})$. Finally, the last two statements follow from linearity of $\overline{\mathrm{Pr}}$, Theorem 1.20 and Proposition 2.13.

Remark 2.15. From the defining property of QC̄R and Propositions 2.9, 2.13 we see that $\mathrm{Q} \bar{C} R(\nabla f)=\bar{\nabla} f$ for every $f \in \operatorname{Test}(\mathrm{X})$. Then it is easy to see that $\mathrm{Q} \bar{C} \mathrm{R}(\operatorname{Test} \mathrm{V}(\mathrm{X}))=\operatorname{Test} \overline{\mathrm{V}}(\mathrm{X})$. 
Remark 2.16 (Alternative notion of quasi-continuous vector field). It is well-known that a vector field $v: \mathbb{R}^{n} \rightarrow \mathbb{R}^{n}$ in the Euclidean space is quasi-continuous if and only if $\mathbb{R}^{n} \ni x \mapsto|v(x)-\nabla f(x)|$ is quasi-continuous for every smooth function $f: \mathbb{R}^{n} \rightarrow \mathbb{R}$. This would suggest an alternative definition of quasi-continuous vector field on the $\operatorname{RCD}(K, \infty)$ space $\mathrm{X}$ :

$$
\widetilde{\mathcal{Q C}}(T \mathrm{X}):=\left\{v \in L_{\text {Cap }}^{0}(T \mathrm{X})|| v-\bar{\nabla} f \mid \in \mathcal{L} \mathcal{C}(\mathrm{X}) \text { for every } f \in \operatorname{Test}(\mathrm{X})\right\} .
$$

The well-posedness of the previous definition follows from the fact that quasi-continuity is preserved under modification on Cap-negligible sets. As we are going to show, it holds that

$$
\mathrm{Q} \mathcal{C}(T \mathrm{X}) \subset \widetilde{\mathcal{Q}}(T \mathrm{X}) .
$$

In order to prove it, let us fix $v \in \mathcal{Q C}(T \mathrm{X})$. Given any $f \in \operatorname{Test}(\mathrm{X})$ and $\left(v_{n}\right)_{n} \subset \operatorname{Test} \overline{\mathrm{V}}(\mathrm{X})$ such that $v_{n} \rightarrow v$ in $L_{\text {Cap }}^{0}(T \mathrm{X})$, we see (by arguing as in the proof of Lemma 2.12) that $\left|v_{n}-\bar{\nabla} f\right| \in \mathcal{Q} \mathcal{C}(\mathrm{X})$ holds for every $n \in \mathbb{N}$, therefore also $|v-\bar{\nabla} f| \in \mathcal{L} \mathcal{C}(\mathrm{X})$ as an immediate consequence of the fact that $\left|v_{n}-\bar{\nabla} f\right| \rightarrow|v-\bar{\nabla} f|$ in $L^{0}(\mathrm{Cap})$. Since $f \in \operatorname{Test}(\mathrm{X})$ is arbitrary, the claim (2.24) is proven.

Notice that due to the non-linearity of the defining condition $(2.23)$ it is not clear if $\widetilde{\mathcal{Q C}}(T \mathrm{X})$ is a vector space or not. In particular, it is not clear if the inclusion in (2.24) can be strict.

We conclude giving a simple and explicit example to which the definitions and constructions presented in the paper can be applied:

Example 2.17 (The case $\mathrm{X}=[0,1]$ ). Let us see how the definitions we gave work in the case $\mathrm{X}$ is the Euclidean segment $[0,1]$ equipped with its natural distance and measure. It is well known and easy to check that in this space every singleton has positive capacity. It follows that the space $L^{0}(\mathrm{Cap})$ coincides, as a set, with the space of all real valued Borel functions on $\mathrm{X}$ and similarly the space $\mathcal{L C}(\mathrm{X})$ coincides with the space of continuous functions on $\mathrm{X}$. In particular, the quasi-continuous representative of a Sobolev function is, in fact, its continuous representative.

A direct verification of the definitions then shows that for $f \in D(\Delta) \subset W^{1,2}(\mathrm{X})$ we have $f^{\prime} \in W^{1,2}(\mathrm{X})$ as well and, identifying $f, f^{\prime}$ with their continuous representatives, it also holds $f^{\prime}(0)=f^{\prime}(1)=0$. In particular, for any $f \in \operatorname{Test}(\mathrm{X})$ we have that (the continuous representative of) $f^{\prime}$ is continuous and equal to 0 in $\{0,1\}$.

We now claim that $L_{\text {Cap }}^{0}(T X)$ is (=can be identified with) the space of Borel functions on $[0,1]$ which are 0 on $\{0,1\}$, the corresponding gradient map $\bar{\nabla}$ being the one which assigns to any $f \in \operatorname{Test}(\mathrm{X})$ the continuous representative of $f^{\prime}$, which shall hereafter be denoted by $f^{\prime}$. The verification of this claim follows from the above discussion and the uniqueness part of Theorem 2.6 .

It is then clear that $\mathcal{Q}(T \mathrm{X})$ consists of continuous elements in $L_{\text {Cap }}^{0}(T \mathrm{X})$, i.e. of continuous functions which are zero on $\{0,1\}$, and that $\mathcal{Q} \mathcal{C}(T \mathrm{X})$ coincides with the space $\widetilde{\mathcal{Q C}}(T \mathrm{X})$ introduced in the previous remark.

This simple example shows that:

a) The constant dimension property of $\operatorname{RCD}(K, N)$ spaces recently obtained in [5], which is known to carry over to the 'standard' tangent module $L_{\mathfrak{m}}^{0}(T \mathrm{X})$, does not carry over to the module $L_{\text {Cap }}^{0}(T X)$ introduced in this manuscript: adapting the definitions in [9], one can see that in our example the dimension of $L_{\text {Cap }}^{0}(T X)$ over $\{0,1\}$ is 0 and over $(0,1)$ is 1 .

b) The estimates obtained in [14] from which one can deduce that the capacity of the critical set of solutions of elliptic PDEs is 0, do not carry over to the RCD setting, and in fact not even in the setting of non-collapsed RCD spaces. Indeed, in our example the critical set of any function on $\mathrm{X}$ whose Laplacian is also a function (and not a measure) contains 
$\{0,1\}$ : the problem seems to be the presence of the 'boundary', see also [11] for further comments about the definition of boundary of a ncRCD space.

\section{REFERENCES}

[1] L. Ambrosio, N. Gigli, And G. Savaré, Density of Lipschitz functions and equivalence of weak gradients in metric measure spaces, Rev. Mat. Iberoam., 29 (2013), pp. 969-996.

$[2]-$ Calculus and heat flow in metric measure spaces and applications to spaces with Ricci bounds from below, Invent. Math., 195 (2014), pp. 289-391.

[3] A. BJöRn AND J. BJöRN, Nonlinear potential theory on metric spaces, vol. 17 of EMS Tracts in Mathematics, European Mathematical Society (EMS), Zürich, 2011.

[4] N. Bouleau And F. Hirsch, Dirichlet forms and analysis on Wiener space, De Gruyter studies in mathematics, W. de Gruyter, 1991.

[5] E. Brù̀ And D. Semola, Constancy of the dimension for $\operatorname{RCD}(K, N)$ spaces via regularity of Lagrangian flows. Accepted at Comm.Pure and Appl.Math., arXiv: 1804.07128.

[6] J. Cheeger, Differentiability of Lipschitz functions on metric measure spaces, Geom. Funct. Anal., 9 (1999), pp. $428-517$.

[7] G. De Philippis And J. NúÑeZ-Zimbrón, work in progress. Preprint.

[8] D. Denneberg, Non-Additive Measure and Integral, Theory and Decision Library B, Springer Netherlands, 2010.

[9] N. GigLI, Nonsmooth differential geometry - an approach tailored for spaces with Ricci curvature bounded from below, Mem. Amer. Math. Soc., 251 (2014), pp. v+161.

[10] — Lecture notes on differential calculus on RCD spaces, (2018). Publ. RIMS Kyoto Univ. 54.

[11] N. Gigli And G. De Philippis, Non-collapsed spaces with Ricci curvature bounded from below. Accepted at J. Ec. Polyt., arXiv:1708.02060.

[12] J. Heinonen, P. Koskela, N. Shanmugalingam, and J. T. Tyson, Sobolev spaces on metric measure spaces. An approach based on upper gradients, vol. 27 of New Mathematical Monographs, Cambridge University Press, Cambridge, 2015.

[13] P. Koskela, K. Rajala, and N. Shanmugalingam, Lipschitz continuity of Cheeger-harmonic functions in metric measure spaces, J. Funct. Anal., 202 (2003), pp. 147-173.

[14] A. Naber And D. Valtorta, Volume estimates on the critical sets of solutions to elliptic PDEs, Comm. Pure Appl. Math., 70 (2017), pp. 1835-1897.

[15] G. Savaré, Self-improvement of the Bakry-Émery condition and Wasserstein contraction of the heat flow in $\operatorname{RCD}(K, \infty)$ metric measure spaces, Discrete Contin. Dyn. Syst., 34 (2014), pp. 1641-1661.

[16] N. Shanmugalingam, Newtonian spaces: an extension of Sobolev spaces to metric measure spaces, Rev. Mat. Iberoamericana, 16 (2000), pp. 243-279.

Institut Polytechnique de Grenoble (CPP - La Prépa des INP), Domaine universitaire, 701 rue de la PISCINE, 38402 Saint Martin D'HÈres.

E-mail address: clement.debin@gmail.com

SiSSA, Via Bonomea 265, 34136 Trieste

E-mail address: ngigli@sissa.it

Department of Mathematics and Statistics, P.O. Box 35 (MaD), FI-40014 University of Jyväskylä

E-mail address: enpasqua@jyu.fi 